\title{
Efficient Near-Field to Mid-Field Sonic Boom Propagation using a High-Order Space Marching Method
}

\author{
Jeffrey A. Housman, Gaetan K. Kenway† James C. Jensen $\ddagger$ and Cetin C. Kiris ${ }^{\S}$
}

NASA Ames Research Center, M/S 258-2, Moffett Field, CA 94035

\begin{abstract}
An efficient strategy for propagating sonic boom signatures from a near-field Computational Fluid Dynamics (CFD) solution to the mid-field is presented. The method is based on a high-order accurate finite-difference discretization of the $3 \mathrm{D}$ Euler equations on a specially designed curvilinear grid and a single sweep space marching solution algorithm. The new approach leads to more than a factor of two reduction in overall computational resources compared to the current method used to propagate near-field sonic booms to the ground. Accuracy and efficiency of the near-field to mid-field process is demonstrated using a selection of test cases from the AIAA Sonic Boom Prediction Workshops. Azimuthal dependence of nonlinear wave propagation from the near-field to mid-field is analyzed along with its effects on the ground level noise.
\end{abstract}

\section{Introduction}

As NASA's Low-Boom Flight Demonstration (LBFD) project matures, there is an increasing need for accurate and efficient computational prediction tools for estimating the primary boom carpet and associated ground level noise footprint. The X-59 Quiet Supersonic Technology (QueSST) airplane, is a 94 foot-long, 29.5 foot wide, single jet engine aircraft, designed to fly at Mach 1.42 at 55,000 feet, and is targeted for completion by $2021 .{ }^{1}$ One of the primary goals of the project is to demonstrate the feasibility of supersonic over-land flight at reduced loudness levels. Computational tools will be heavily relied on for mission planning of the low-boom flight tests. These tools must be accurate, efficient, and easy to use.

The current approach for predicting the ground level noise of a supersonic aircraft consists of a two step process. In the first step, Computational Fluid Dynamics (CFD) is used to solve the supersonic flow around the aircraft, which is denoted the "near-field" solution. This is typically done using second order accurate numerical methods on unstructured, structured overlapping, or Cartesian grids. ${ }^{2-5}$ The second step of the process propagates the pressure traces extracted from the near-field solution to the ground using a combination of geometrical acoustic ray tracing and a lossy Burgers equation. ${ }^{6-8}$ The current far-field propagation methods assume that the azimuthal dependence of the near-field solution is insignificant to the wave propagation of the extracted pressure trace. This approach is only accurate when the radial distance at which the near-field solution is extracted is "far enough" away from the aircraft. Supersonic flows around advanced low-boom aircraft designs, such as the X-59, include wings, nacelles, and propulsion systems which generate potentially non-negligible azimuthal variations for several body lengths (from 6-10 body lengths or more) in the radial direction. ${ }^{9,10}$ With the current approach, the near-field CFD domain must be extended and a relatively fine grid has to be used to capture the small but important acoustic wave variations. The additional mesh resolution pushes the CPU cost of a simulation significantly higher than required for aerodynamic performance calculations, which becomes prohibitive when a large database of solutions are being generated for mission planning.

\footnotetext{
*Computational Aerosciences Branch, AIAA Senior Member, jeffrey.a.housman@nasa.gov

†Science and Technology Corporation, AIAA Member, gaetan.k.kenway@nasa.gov

${ }^{\ddagger}$ Computational Aerosciences Branch, AIAA Member, james.c.jensen@nasa.gov

$\S$ Computational Aerosciences Branch, AIAA Senior Member, cetin.c.kiris@nasa.gov
} 
An alternative approach is to take advantage of the supersonic nature of the flow field by using a specialized grid and numerical method known as space marching. This method can significantly reduce the computational cost of the propagation compared to traditional CFD, while maintaining the accuracy. The new method can be introduced as an intermediate step between the near-field CFD solution and the far-field propagation processes. The near-field CFD will still be responsible for resolving the flow-field around the aircraft, but the radial extent of the far-field boundary can be significantly truncated to less than one body length. The near-field CFD solution can then be interpolated onto the initial layers of the space marching grid at a radial location slightly larger than the wing semi-span, and propagated (space-marched) until the azimuthal dependence becomes negligible. This will occur before atmospheric effects must be accounted for and will be denoted the "mid-field" in this work. From there, the pressure can be propagated to the ground using the existing far-field wave propagation tools. The space marching process, including grid generation, interpolation of the CFD solution, and performing the single sweep space marching algorithm can be completed in minutes on a high-end workstation as opposed to hours for a mid-field CFD simulation on a supercomputer. Furthermore, truncation of the radial extent of the CFD mesh can significantly reduce the number of grid points (somewhere between 20-40 percent or more) to a level commensurate with standard aerodynamic performance calculations. This proportionally reduces the computational cost of the CFD solution through a combination of reducing the necessary number of CPU cores required and improving the iterative convergence of the solver.

Two distinct approaches have been reported in the literature for the intermediate step of propagating the nonlinear pressure waves from the near-field to the mid-field. The first approach utilizes a multipole matching procedure ${ }^{11-14}$ based on the expansion derived by George. ${ }^{15}$ This method relies on projecting the near-field CFD solution onto a sequence of multipole distributions representing the Whitham F-function. ${ }^{16}$ Then at each azimuth, the far-field F-function representation is used as input into the far-field propagation code. The success of this approach not only relies on the accuracy of the multipole expansion, but also on the procedure which projects the near-field CFD solution onto the multipole basis. The second method utilizes the Euler equations (or reduced forms) directly, including the full-potential equation, ${ }^{17,18}$ or a space marching approach. ${ }^{19-21}$ It is the space marching method that is explored in this work.

Space marching is a numerical technique applied to the Euler or Parabolized Navier-Stokes equations developed in the $1970{ }^{\prime 22-24}$ as an efficient solution procedure for three-dimensional supersonic flow with small regions of subsonic flow near solid boundaries. By marching, either explicitly or implicitly, in the streamwise direction, the three-dimensional problem is reduced to an unsteady-like two-dimensional one with significant savings in both storage and runtime. In the 1980's, the method was extended to flows with subsonic pockets, ${ }^{25}$ equilibrium air computations around hypersonic vehicles, ${ }^{26}$ and upwind total variation diminishing (TVD) algorithms. ${ }^{27}$ As high-performance computing resources became more widely available and distributed memory machines became affordable, time-marching methods became more attractive since a dominant flow direction is not necessary for steady-state convergence. Nevertheless, some groups have continued to mature space marching methods ${ }^{28-31}$ because of their fast convergence properties for predominantly supersonic flow fields.

A high-order accurate space marching solver has been developed for the Launch Ascent and Vehicle Aerodynamics (LAVA) framework. ${ }^{32}$ The method is closely related to the work of Shen and Lazzaro, ${ }^{21}$ but utilizes a specialized orthogonal Mach-cone aligned curvilinear grid and an implicit solution procedure for greater efficiency. This approach significantly reduces the required streamwise and radial grid resolution requirements necessary to achieve accurate mid-field pressure signatures when compared to orthogonal cylindrical grids, such as those used in Shen and Lazzaro. ${ }^{21}$ The computational methodology is described in Section II including the governing equations, specialized curvilinear grid, numerical discretization, and implicit solution procedure. Results from the application of the space marching algorithm are presented in Section III. First, a sequence of grid and solver parameter studies are performed on the JAXA Wing Body (JWB) test case from the Second AIAA Sonic Boom Prediction Workshop. Sensitivity analysis of the grid and solver parameters is used to assess a baseline set of parameters appropriate for general use. Next, a detailed analysis of the azimuthal dependence of nonlinear wave propagation is performed for the JWB. Finally, using the best practices developed for the JWB, the space marching method is applied to a more realistic low boom aircraft design, the Lockheed Martin 1021 (LM-1021) test case from the First AIAA Sonic Boom Prediction workshop. Results from the application of the space marching method to the LM-1021 are compared to existing CFD results as well as experimental wind tunnel results. A summary of the work is discussed in Section IV along with some closing remarks on how the current method maybe extended to 
propagate the near-field signatures directly to the ground.

\section{Computational Methodology}

Nonlinear wave propagation from supersonic aircraft fits well within the context of space marching methods. The high-order accurate space marching algorithm described here was developed as an additional solver for the LAVA framework. The space marching solver utilizes similar high-order finite-difference methods and overset grid interpolation routines as those used in the curvilinear CFD solver in LAVA. Note, tecplot can be used for the interpolation procedure for CFD solutions on non-overlapping grids, such as unstructured or Cartesian grids. First, the governing equations are presented. Next, the specially designed structured curvilinear, Mach-cone aligned grid generation procedure is described. Then the numerical discretization of the governing equations and the implicit space marching solution procedure is outlined.

\section{II.A. Governing Equations}

The equations governing the evolution of the near-field supersonic flow to the mid-field are the steady-state Euler equations of gas dynamics. The definition of near-field to mid-field used in this work can be described as the cylindrical region around the aircraft with a starting radius of approximately 0.5 to 1.5 span lengths and extending to a radial distance of approximately 10 body lengths from the aircraft. Atmospheric effects, such as hydrostatic balance and thermal stratification, are still negligible in this range. The steady Euler equations can be written for a general curvilinear coordinate system in strong conservation law form ${ }^{33}$ as,

$$
\frac{\partial \hat{E}}{\partial \xi}+\frac{\partial \hat{F}}{\partial \eta}+\frac{\partial \hat{G}}{\partial \zeta}=0
$$

where

$$
\hat{E}=\left[\begin{array}{c}
\rho \hat{U} \\
\rho \hat{U} u+p \hat{\xi}_{x} \\
\rho \hat{U} v+p \hat{\xi}_{y} \\
\rho \hat{U} w+p \hat{\xi}_{z} \\
\rho \hat{U} H
\end{array}\right], \hat{F}=\left[\begin{array}{c}
\rho \hat{V} \\
\rho \hat{V} u+p \hat{\eta}_{x} \\
\rho \hat{V} v+p \hat{\eta}_{y} \\
\rho \hat{V} w+p \hat{\eta}_{z} \\
\rho \hat{V} H
\end{array}\right], \hat{G}=\left[\begin{array}{c}
\rho \hat{W} \\
\rho \hat{W} u+p \hat{\zeta}_{x} \\
\rho \hat{W} v+p \hat{\zeta}_{y} \\
\rho \hat{W} w+p \hat{\zeta}_{z} \\
\rho \hat{W} H
\end{array}\right] .
$$

Standard notation is used where $\rho$ is the density, $p$ the pressure, $(u, v, w)$ the Cartesian velocity components, and $H=h+\frac{1}{2}\left(u^{2}+v^{2}+w^{2}\right)$ is the total enthalpy. The contravariant velocities in the $(\xi, \eta, \zeta)$ coordinate directions are denoted

$$
\hat{U}=u \hat{\xi}_{x}+v \hat{\xi}_{y}+w \hat{\xi}_{z}, \quad \hat{V}=u \hat{\eta}_{x}+v \hat{\eta}_{y}+w \hat{\eta}_{z}, \quad \hat{W}=u \hat{\zeta}_{x}+v \hat{\zeta}_{y}+w \hat{\zeta}_{z}
$$

Note the metric relations have been scaled by the Jacobian of the metric transformation

$$
\left(\hat{\xi}_{x}, \hat{\xi}_{y}, \hat{\xi}_{z}\right)=J^{-1}\left(\xi_{x}, \xi_{y}, \xi_{z}\right), \quad\left(\hat{\eta}_{x}, \hat{\eta}_{y}, \hat{\eta}_{z}\right)=J^{-1}\left(\eta_{x}, \eta_{y}, \eta_{z}\right), \quad\left(\hat{\zeta}_{x}, \hat{\zeta}_{y}, \hat{\zeta}_{z}\right)=J^{-1}\left(\zeta_{x}, \zeta_{y}, \zeta_{z}\right) .
$$

The equation set is closed by the ideal gas law which relates $p=\rho R T$, where $R=C_{p}(\gamma-1) / \gamma, C_{p}$ is the specific heat at constant pressure, and $\gamma$ is the ratio of specific heats.

\section{II.B. Mach-cone Aligned Space Marching Grid}

A specially designed structured curvilinear grid is used to discretize the space between the near-field and mid-field. Structured curvilinear grids have several advantages over unstructured, structured cylindrical, and Cartesian grids for this particular application. The advantages over unstructured grids include the high efficiency and low memory footprint of the corresponding numerical algorithm and the straightforward and relatively inexpensive extension to high-order low-dissipation finite-difference discretizations which are the most efficient for weakly nonlinear wave propagation. The main advantage over structured cylindrical and Cartesian grids are the ability to create a Mach-cone aligned mesh for each azimuth with a single grid. The advantage of using Mach-cone aligned grids for nonlinear wave propagation in low boom environments has been shown by several groups. ${ }^{34,35}$ One of the first publications demonstrating the use of Mach-cone aligned grids was Siclari and Darden, ${ }^{20}$ where they used a cylindrical/spherical coordinate system with 
Mach-cone alignment for the bow shock to capture sonic booms from simplified fuselage wing configurations and propagate them to the mid-field. In fact, the advantage of Mach-cone alignment is so great, that when using adjoint based mesh adapted Cartesian grids, it was found to be more efficient to solve for a single azimuth of interest (or a tight range) independently, while insuring Mach-cone alignment with respect to that azimuth, than to try and solve for all azimuths at once. ${ }^{5}$

Inspired by the grid topology used in Siclari and Darden ${ }^{20}$ and implicit solution strategies used in overset grid methods, a specially designed structured curvilinear, Mach-cone aligned, cylindrical grid generation procedure, which utilizes iblanking overset grid technology, was established. Figure 1 (a) and (b) show the symmetry plane and the final streamwise station of the space marching grid along with a low-boom aircraft (the JAXA wing-body configuration) shown in red. The $(\xi, \zeta)$ plane of the grid, at each $\eta$ (or azimuth), is orthogonal with lines of constant $\xi$ aligned with the free-stream Mach cone angle. The inputs for the grid generation procedure include: $M_{\infty}$ the free-stream Mach number; $\left(\mathbf{x}_{\mathbf{0}}, \mathbf{r}_{\mathbf{0}}, \theta_{\mathbf{0}}\right)$ the starting axial, radial, and circumferential locations of the inner cylinder surface; $\left(\mathbf{L}, \mathbf{R}, \theta_{\max }\right)$ the axial length, radial extent, and circumferential extent of the mesh; $\mathbf{N}_{\mathbf{S}}$ and $\mathbf{N}_{\theta}$ the number of points in the streamwise axial and circumferential directions; $\mathbf{S R}$ and $\mathbf{A} \mathbf{R}_{\max }$ the stretching ratio and maximum aspect ratio in the space marching direction. Notice that the grid points of the mesh inside a cylinder of radius $r_{0}$ are not shown in the plot, these points are marked as blank points or points which will not be used to solve the system of partial differential equations. This concept of blanking is well-known in the overset grid community and is used here in a novel way to allow an orthogonal grid to be generated with Mach-cone alignment. This allows the space marching direction to be chosen to go out radially from the near-field, as opposed to the streamwise direction used for a standard cylindrical mesh. ${ }^{21}$ This has two clear advantages over the standard cylindrical mesh approach. The first, which was already mentioned, is Mach cone alignment which effectively reduces the artificial dissipation of the method allowing a fewer number of points to be used for a given level of error. The second advantage, which is more subtle, is that the implicit solution is now effectively constrained in the streamwise and circumferential direction, both of which are typically much smaller in dimension compared to the radial extent. This allows space marching to be applied in the radial direction, which becomes the largest dimension in the grid as the radial extraction distance increases.

The grid generation procedure is split-up into several simple steps. Figure 2 (a) shows the beginning step which consists of generating a rectangle on the $x-z$ symmetry plane with diagonal length $L$, the axial extent of the inner cylinder, and an inner acute angle $\mu=\sin ^{-1}\left(1 / M_{\infty}\right)$ which is the Mach cone angle. Next, the rectangle is discretized using a spacing of $\Delta x=\Delta s \cos (\mu)$ and $\Delta z=\Delta s \sin (\mu)$, where $\Delta s=L /\left(N_{\text {strm }}-1\right)$ (Figure $2(\mathrm{~b})$ ). An extension length is then added to the rectangle in the positive $x$ direction (Figure $2(\mathrm{c})$ ). The length of the extension is $\left(R-r_{0}\right) / \sin (\mu)$, where $R$ is the radial extent of the space marching grid. During this extension procedure, the mesh spacing is stretched using the input stretching ratio, $S R$, until the aspect ratio reaches $A R_{\max }$, at which point the mesh spacing in the extension direction remains fixed. Now that the size and spacing of the mesh has been established on the symmetry plane, the grid is rotated about the $y$-axis by the Mach-cone angle $\mu$ plus a small perturbation $\mu_{\epsilon}$ resulting in a nearly Mach-cone aligned mesh for the on-track signature (Figure $2(\mathrm{~d})$ ). The small perturbation is used to reduce the chance of the numerical flux being applied across a sonic-line, where many numerical fluxes generate spurious oscillations. The symmetry plane grid is also translated such that the origin of the rectangle starts at $\left(x_{0}, r_{0}\right)$ (Figure 2 (e)). Now the grid is ready for the blanking to be applied. The blanking procedure consists of marking all of the points with a $z$ coordinate above $-r_{0}$ with an iblank value of 0 . This is shown in Figure 2 (f) along with the low-boom aircraft for reference. The points above the blue line have been greyed out to represent the blanking. Plotting of these points is suppressed in the subsequent plots, as is typically done for overset grids. The symmetry plane mesh is now ready to be rotated to the $\theta_{0}$ position and revolved from $\theta_{0}$ to $\theta_{\max }$ using $N_{\theta}$ grid points (Figure $2(\mathrm{~g})$ ). Note that without blanking, all of the grid points with $z$ coordinate above 0 would generate negative cell volumes once the symmetry plane is revolved. The final step is to mark the necessary number of fringe layers (interpolation points) required for the spatial discretization used in the space marching direction. For a second order accurate scheme in the space marching direction (BDF2) two layers of fringe points are necessary. These are plotted as magenta colored spheres in Figure 2 (h). A final plot of the symmetry plane of the space marching grid along with the CFD grid is shown in Figure 3, illustrating the inner cylinder of the space marching grid is contained with the CFD grid for proper solution interpolation. 


\section{II.C. Numerical Discretization}

Now that the structured grid and associated curvilinear coordinate directions are established, the numerical discretization procedure is described. The space marching direction is defined to be in the $\zeta$-coordinate direction. The choice of using the $\zeta$-coordinate as the space marching direction allows for a cache-friendly implementation on modern computer hardware. The $\xi$-coordinate direction is defined to be orthogonal to the $\zeta$-coordinate direction at a fixed azimuth. Orthogonal grids are known to reduce discretization errors in finite-difference schemes compared to highly skewed grid lines. The $\eta$ coordinate is assigned to the circumferential direction. Two distinct approaches to the derivative approximations are used to discretize the governing equations depending on the coordinate direction. In the space marching direction, backward differencing formulas are utilized to discretize the flux derivative. These are typically used for discretizing the time-derivative in unsteady Navier-Stokes calculations, and are a natural choice for the space marching direction since the waves can only travel in this direction on the specially designed grid. Two different orders of accuracy for backward differencing are implemented in the current space marching solver; first-order and second-order

$$
\begin{aligned}
\frac{\partial \hat{G}}{\partial \zeta} & \approx \frac{\hat{G}_{l}-\hat{G}_{l-1}}{\Delta \zeta} \text { (first-order) } \\
\frac{\partial \hat{G}}{\partial \zeta} & \approx \frac{3\left(\hat{G}_{l}-\hat{G}_{l-1}\right)-\left(\hat{G}_{l-1}-\hat{G}_{l-2}\right)}{2 \Delta \zeta} \text { (second-order). }
\end{aligned}
$$

In the cross-stream direction, a high-order Hybrid Weighted Compact Nonlinear Scheme (HWCNS) ${ }^{36-38}$ is used to approximate the $(\xi, \eta)$ derivatives. The HWCNS utilizes a central difference stencil that combines numerical fluxes at the edges with physical fluxes at the nodes resulting in high spectral accuracy, which is important for weakly nonlinear wave propagation. An example of the HWCNS discretization applied to the $\xi$-coordinate convective flux is,

$$
\frac{\partial \hat{E}}{\partial \xi} \approx \frac{a_{1}\left(\tilde{E}_{j+1 / 2}-\tilde{E}_{j-1 / 2}\right)+a_{2}\left(\hat{E}_{j+1}-\hat{E}_{j-1}\right)+a_{3}\left(\hat{E}_{j+2}-\hat{E}_{j-2}\right)}{\Delta \xi} .
$$

The coefficients $a_{1}, a_{2}$, and $a_{3}$ can be chosen to obtain either second (HWCNS2), fourth (HWCNS4), or sixth (HWCNS6) order accuracy. The step-sizes $\Delta \xi$ and $\Delta \zeta$ have been taken to be unity. A similar formula is available for the $\eta$-coordinate derivative. The artificial dissipation in the scheme is introduced through the numerical fluxes at the edges, $\tilde{E}_{j+1 / 2}\left(Q_{L}, Q_{R}\right)$, which are constructed with a modified Roe numerical flux ${ }^{39-41}$ with left and right state interpolations, $Q_{L}$ and $Q_{R}$, generated from a high-order WENO interpolation. ${ }^{42}$ In the current implementation, third and fifth order accurate upwind biased WENO interpolations (WENO3 and WENO5), an improved fifth-order accurate WENO interpolation ${ }^{43}$ (ZWENO5), as well as the optimal weight counterparts (OPT3 and OPT5) are available.

In order to reduce any unnecessary artificial dissipation generated from the WENO interpolations, and retain the spectral resolution of the HWCNS, a blending of pure central interpolation with the upwind biased interpolation can be used. The blending factor between central and upwind biased interpolation is based on the local contravariant Mach number in each cross-stream direction at the half point. This is an improvement over the standard local Mach number blending used previously. ${ }^{44-46}$

$$
\begin{aligned}
Q_{L} & =\frac{1}{2}\left(Q_{L}^{\mathrm{WENO}}+Q_{R}^{\mathrm{WENO}}\right)+\frac{\beta}{2}\left(Q_{L}^{\mathrm{WENO}}-Q_{R}^{\mathrm{WENO}}\right) \\
Q_{R} & =\frac{1}{2}\left(Q_{L}^{\mathrm{WENO}}+Q_{R}^{\mathrm{WENO}}\right)+\frac{\beta}{2}\left(Q_{R}^{\mathrm{WENO}}-Q_{L}^{\mathrm{WENO}}\right),
\end{aligned}
$$

where

$$
\beta=\min \left(\max \left(\frac{\hat{U}_{L}}{\hat{c}_{L}}, \frac{\hat{U}_{R}}{\hat{c}_{R}}, \beta_{\min }\right), 1\right),
$$

$\hat{U}$ is the contravariant velocity, $\hat{c}=\sqrt{\gamma R T\left(\hat{\xi}_{x}^{2}+\hat{\xi}_{y}^{2}+\hat{\xi}_{z}^{2}\right)}$ is the metric scaled sound speed in the $\xi$-coordinate direction, and $0 \leq \beta_{\min } \leq 1$. Typical values for $\beta_{\min }$ are between 0 and 0.1 . Similar blending functions are used in the $\eta$-coordinate direction. If third (or fifth) order optimal weights are chosen for the WENO 
interpolation and $\beta=0$, then central interpolations of fourth (or sixth) order are returned from the blending. For non-optimal weights, or $\beta>0$, upwind biased interpolations are returned, and the order of accuracy returns to third (or fifth) but the magnitude of the artificial dissipation is reduced. The blending parameter, $\beta_{\text {min }}$, must be chosen with care since too low of a value can lead to non-physical oscillations and potential solution divergence.

It is well known that free-stream preservation (i.e. the GCL condition) requires that identical finitedifference operators are used to compute the metric terms, as those used to discretize the convective flux derivatives. ${ }^{47-49}$ The derivatives which appear in the metric term evaluations for the $(\xi, \eta)$-coordinate directions utilize the same HWCNS as that used for the convective flux where the required half-point grid values are obtained from central interpolations of the same order as the derivative evaluation. In the space marching direction, the metric terms are evaluated with the BDF formulas above. Evaluating the metric terms using standard central differencing, or using the HWCNS in the space marching direction leads to a breakdown in free-stream preservation. Examples of this will be shown in the results section.

To summarize, the nonlinear discrete residual operator at an arbitrary space marching $\zeta$ surface $l$, and $(\xi, \eta)$ grid point location location $(j, k)$, is written as,

$$
\begin{aligned}
R_{j, k, l} & =a_{1}\left(\tilde{E}_{j+1 / 2}-\tilde{E}_{j-1 / 2}\right)+a_{2}\left(\hat{E}_{j+1}-\hat{E}_{j-1}\right)+a_{3}\left(\hat{E}_{j+2}-\hat{E}_{j-2}\right) \\
& +a_{1}\left(\tilde{F}_{k+1 / 2}-\tilde{F}_{k-1 / 2}\right)+a_{2}\left(\hat{F}_{k+1}-\hat{F}_{k-1}\right)+a_{3}\left(\hat{F}_{k+2}-\hat{F}_{k-2}\right) \\
& +\left(\delta_{\zeta}^{(b)} \hat{G}\right)_{l}
\end{aligned}
$$

This represents a system of nonlinear equations, on the surface of constant $l$, which must be iteratively solved. Once the system is solved on this surface, the solution on the next surface, $l+1$, can be found. An efficient implicit solution procedure is described next.

\section{II.D. Implicit Solution Procedure}

In the single sweep space marching procedure, the solution is marched in the $\zeta$-coordinate direction starting from an interpolated CFD solution on the inner cylinder of fringe points, $Q_{r=r_{0}}^{\text {fringe }}=\operatorname{Interp}\left(Q_{C F D}\right)$. At each new streamwise station, the nonlinear system of equations is solved on the $(\xi, \eta)$ plane with the evaluation of $\hat{G}_{l-1}$ and $\hat{G}_{l-2}$ set from the previous $(\xi, \eta)$ plane solutions. Only a single sweep is necessary provided the contravariant Mach number in the streamwise direction is larger than one, $\hat{M}_{\zeta}=\hat{W} / \hat{c}>1$, for all streamwise stations. This condition is satisfied when using the specially designed structured curvilinear grid and results in a reduced nonlinear system of equations where only the solution of the current plane $Q_{l}$ is unknown for all $(j, k)$. Introducing the iteration parameter, $m$, and assuming a first-order discretization in the cross-marching directions, the system at the next $l+1$ plane can be represented as,

$$
\begin{aligned}
\mathcal{R}\left(Q_{m+1}, Q_{l}, Q_{l-1}\right) & =\left(\tilde{E}_{j+1 / 2, m+1}-\tilde{E}_{j-1 / 2, m+1}\right)+\left(\tilde{F}_{j+1 / 2, m+1}-\tilde{F}_{j-1 / 2, m+1}\right) \\
& +\frac{3}{2}\left(\hat{G}_{m+1}-\hat{G}_{l}\right)-\frac{1}{2}\left(\hat{G}_{l}-\hat{G}_{l-1}\right) .
\end{aligned}
$$

Now each term depending on $m+1$ can be linearized about the previous iteration (on the current $l+1$ plane),

$$
\begin{aligned}
\tilde{E}_{j+1 / 2, m+1} & \approx \tilde{E}_{j+1 / 2, m}+\left(\frac{\partial \tilde{E}_{j+1 / 2, m}}{\partial Q_{j+1, m}}\right) \Delta Q_{j+1, m}+\left(\frac{\partial \tilde{E}_{j+1 / 2, m}}{\partial Q_{j, m}}\right) \Delta Q_{j, m} \\
\tilde{F}_{k+1 / 2, m+1} & \approx \tilde{F}_{k+1 / 2, m}+\left(\frac{\partial \tilde{F}_{k+1 / 2, m}}{\partial Q_{k+1, m}}\right) \Delta Q_{k+1, m}+\left(\frac{\partial \tilde{F}_{k+1 / 2, m}}{\partial Q_{k, m}}\right) \Delta Q_{k, m} \\
\hat{G}_{m+1} & \approx \hat{G}_{m}+\left(\frac{\partial \hat{G}_{m}}{\partial Q_{m}}\right) \Delta Q_{m} .
\end{aligned}
$$


Using the above linearization and introducing a pseudo-time stepping term to improve convergence during the early stages of the nonlinear planar relaxation procedure, Equation 12 can be rewritten as,

$$
\begin{aligned}
& -\left(\frac{\partial \tilde{F}_{k-1 / 2, m}}{\partial Q_{k-1, m}}\right) \Delta Q_{k-1, m}-\left(\frac{\partial \tilde{E}_{j-1 / 2, m}}{\partial Q_{j-1, m}}\right) \Delta Q_{j-1, m} \\
& +\left[\frac{3}{2} \frac{\Gamma}{J \Delta \tau}+\left(\frac{\partial \tilde{E}_{j+1 / 2, m}}{\partial Q_{j, m}}\right)-\left(\frac{\partial \tilde{E}_{j-1 / 2, m}}{\partial Q_{j, m}}\right)+\left(\frac{\partial \tilde{F}_{k+1 / 2, m}}{\partial Q_{k, m}}\right)-\left(\frac{\partial \tilde{F}_{k-1 / 2, m}}{\partial Q_{k, m}}\right)\right] \Delta Q_{j, k, m} \\
& +\left(\frac{\partial \tilde{E}_{j+1 / 2, m}}{\partial Q_{j+1, m}}\right) \Delta Q_{j+1, m}+\left(\frac{\partial \tilde{F}_{k+1 / 2, m}}{\partial Q_{k+1, m}}\right) \Delta Q_{k+1, m} \\
& =-\mathcal{R}\left(Q_{m}, Q_{l}, Q_{l-1}\right),
\end{aligned}
$$

where $\Gamma=\partial W / \partial Q$ is the change of variables matrix from conservative variables $W$ to primitive variables $Q$. Replacing the right-hand-side, $\mathcal{R}$, with the high-order accurate discretization, $R$ in Equation 10, results in a sparse block linear system of equations. Blanked points are handled by replacing the left-hand-side blocks by the identity matrix on the diagonal and setting the right-hand-side to zero. This decouples the blanked points from the system of equations. Equation 13 is approximately solved to obtain a quasi-Newton correction $\Delta Q_{m}$. As the iteration procedure converges $m \rightarrow \infty$, the norm of the high-order residual converges and the nonlinear system of equations at that plane is satisfied $\left(\|R\| \rightarrow 0\right.$ and $\left.Q_{m} \rightarrow Q_{l+1}\right)$. In the current implementation an alternating line-Jacobi relaxation procedure in the $(\xi, \eta)$-coordinate directions is used. An ILU preconditioned GMRES linear solver is currently being evaluated, but is not included in the current results.

\section{II.E. Near-Field to Mid-Field Propagation}

A diagram of the proposed near-field to mid-field sonic boom propagation procedure is shown in Figures 4 (a)(d). First the near-field CFD grid is generated with a radial extent slightly larger than the semi-span of the aircraft (a reduction of 20-50 percent in total number of grid points compared to the full radial extent CFD mesh). Then the steady-state Reynolds Averaged Navier-Stokes (RANS) CFD solution is computed on the near-field grid. Next, the CFD solution is interpolated onto the fringe points of the space marching grid. For structured overlapping grid systems, an MPI code was developed which uses the same interpolation routines used in overset connectivity to perform this step. For CFD solutions using unstructured or Cartesian grids, Tecplot can be used to interpolate the CFD solution onto the fringe points. Finally, the single sweep space marching procedure is applied to propagate the near-field solution to the mid-field. Note, if the boundary condition used at the radial extent of the CFD mesh is reflecting, then the radial extent of the CFD mesh must be large enough such that the inner cylinder of the space marching grid does not intersect a Mach-cone centered at any upstream node of the radial boundary. Alternatively, a non-reflecting boundary could also be implemented into the CFD solver. We have had no issues with reflections using a Riemann invariant boundary condition with a Mach-cone aligned grid in LAVA CFD simulations using structured curvilinear overset grids.

Some care must be taken when defining the hole cutting inner cylinder since the CFD solution utilizes the RANS equation set, and the space marching solution uses the Euler equations. Figure 5 (a) plots the inner cylinder for the JAXA Wing-Body configuration along with an iso-surface of turbulent eddy viscosity ratio $\mu_{T} / \mu_{\infty}=10$. It has been observed that choosing the radius of the cylinder large enough to not intersect this iso-contour level of turbulent eddy viscosity ratio is sufficient to ensure that the solutions between the two different equation sets match in the overlapping region between the inner cylinder surface the outer radial surface of the CFD mesh. Figures 5 (b)-(d) plot contours lines of the CFD (blue) and the space marching solution (red) at constant $X, Y$, and $Z$ slices. The pressure contour lines generated by the CFD

and space marching solvers are indistinguishable in the overlap region, even though the space marching grid is significantly coarser than the CFD mesh and the convective flux discretization in the space marching direction is different from that used in the CFD solver. 


\section{Results}

The high-order single sweep space marching method described above is used to propagate the near-field CFD solution to the mid-field for two different low-boom aircraft geometries from the First and Second AIAA Sonic Boom Prediction Workshops. In this study, the structured curvilinear overlapping grid CFD solver in LAVA is used to generate the near-field CFD solutions. It has been shown, ${ }^{2,50}$ that structured overset grids are a good option for near-field steady-state RANS simulations of supersonic aircraft. The structured overlapping grids used in this work were generated using a combination of Pointwise ${ }^{51}$ and the Chimera Grid Tools (CGT) software package. ${ }^{52}$

A series of grid and solver parameter studies is performed on the JAXA Wing-Body (JWB) configuration from the Second AIAA Sonic Boom Prediction Workshop. The grid parameters include Mach cone perturbation angle, stretching ratio, maximum aspect ratio, streamwise spacing, circumferential spacing, and maximum circumferential domain extent. The solver parameter studies include metric term evaluation, convective flux discretization, and residual convergence tolerance. Sensitivity of the grid and solver parameters are discussed, and baseline "best practice" settings are established. Next, azimuthal dependence of nonlinear wave propagation is investigated using the JWB. It is shown that the strength of the shock/expansion generated by the wing is reduced significantly faster than the $R^{1 / 2}$ decay of a cylindrical acoustic wave. Large azimuthal velocity is observed in this region, indicating a dipole spreading effect caused by the lift of the wing. The consequence of this azimutal dependence results in high sensitivity of the predicted ground level noise to the radial extraction location for far-field acoustic propagation codes. This sensitivity is not reduced until the radial extraction location becomes greater than $r / L_{\text {ref }} \geq 4$. Finally, the space marching method is applied to the Lockheed Martin 1021 wind tunnel model from the First AIAA Sonic Boom Prediction Workshop, and results are compared to existing CFD results as well as experimental wind tunnel results.

\section{III.A. Sensitivity Studies}

Sensitivity analysis of input parameters is performed through a series of grid and solver parameter studies applied to the JWB. The JWB is an $L_{r e f}=38.7 \mathrm{~m}$ long wing-body configuration flying at an altitude of $15.76 \mathrm{~km}$, flight speed of Mach 1.6 and an angle of attack of 2.3 degrees, resulting in a flight Reynolds number of 5.7 million per meter. A CFD mesh refinement study was performed during the workshop and all reported CFD results in this work were generated with the fine CFD mesh.

A sequential approach is taken to perform the grid and solver parameter studies. In this approach, a set of baseline grid and solver parameters were constructed based on preliminary space marching simulations. The baseline grid parameters include a streamwise grid spacing of $\Delta s=1 / 8 \mathrm{~m}\left(\Delta s / L_{r e f}=0.003\right)$, a circumferential spacing of $\Delta \theta=1^{\circ}$, a stretching ratio of $S R=1.05$, a maximum aspect ratio $A R_{\max }=20$, and a circumferential extent of $\theta_{\max }=180^{\circ}$. For the solver, the fourth-order accurate HWCNS with third-order upwind-biased optimal interpolation weights for left and right state interpolation are used in the $(\xi, \eta)$ directions (HWCNS4-OPT3), while second-order backward differencing is used in the space marching direction. The nonlinear residual is converged 8 orders of magnitude at each space marching station using 2 sweeps of alternating line Jacobi relaxation and local time-stepping. The local time-step is set based on an initial CFL $=10$ and a CFL ramping procedure, which increases the local time-step as the residual converges to zero, was used to increase the convergence rate. These parameters are held fixed during the parameter studies, with the exception of the parameter being analyzed. Since the parameters are not independent, we do not claim to have found the "optimal" choice for the parameter values. Moreover, the studies have only been performed on the JWB, and different conclusions may be found for different configurations.

In order to help quantify the sensitivity of a particular parameter, the following discrete function is applied to pressure signatures extracted from the space marching domain,

$$
f(r, \theta)=\frac{1}{N} \sum_{n=1}^{N}\left(\frac{p\left(x_{n}, r, \theta\right)-p_{\infty}}{p_{\infty}}\right)^{2}, \text { where } x_{n}=x_{0}+(n-1) * \Delta s .
$$

Variations of this function caused by changes in a particular parameter indicate a sensitivity to this parameter within the space marching method. A diagram showing the radial and azimuthal locations of the axial line extractions used for the studies is shown in Figure 6. Both "on-track" $r / L_{\text {ref }}=2.55, \theta=0^{\circ}$ and "off-track"

$r / L_{\text {ref }}=2.55, \theta=50^{\circ}$ signatures are examined. These were the furthest radial and bounding circumferential extraction locations requested for the JWB in the workshop, so verified CFD results using LAVA already 
existed for comparisons. First the grid parameters are investigated, followed by the solver parameters. A figure containing four sub-plots is generated for each grid sensitivity study. The first sub-plot (a) shows effect of the parameter on the space marching grid. The second sub-plot (b) plots the entire pressure signature including the fine CFD solution and the space marching solutions for each parameter evaluation. The third sub-plot (c) plots Equation 14 as a function of the parameter values. The function is normalized by the value of Equation 14 using the baseline parameters, which allows the relative change to be examined. The final sub-plot (d) shows a close-up view of the pressure signature over the domain illustrated in the rectangle plotted in sub-plot (b).

\section{III.A.1. Grid Parameters}

The sensitivity to the Mach cone perturbation angle $\mu_{\epsilon}$ is investigated first. It is well-known that many numerical fluxes used in the discretization of the convective flux derivatives behave poorly across the sonic line. Since the HWCNS used in the space marching solver utilizes an approximate Roe-type numerical flux to obtain the half grid point fluxes, some spurious behavior is expected when the Mach cone perturbation angle $\mu_{\epsilon}=0^{\circ}$. Moreover, since third-order accurate optimal weights (i.e. no WENO limiting) are used to interpolate the left and right states, some spurious behavior may persist for $\mu_{\epsilon}>0^{\circ}$. Figure 7 (a) shows the effect of a non-zero Mach cone perturbation angle on the space marching grid, which has the effect of shifting the constant $\xi$ surfaces to be slightly miss-aligned with the exact free-stream Mach cone angle. The pressure signatures in Figure 7 (b) and (d) show that large spurious wave content is generated when $\mu_{\epsilon}=0.4^{\circ}$. This is in contrast to what occurs in standard second-order accurate numerical schemes which become overly dissipative as the grid deviates from Mach cone alignment. This can be rectified in the current space marching method by turning on the WENO weights, which remove the spurious wave content, but can lead to sub-iteration convergence difficulties (which will be explored in the next sub-section). Spurious wave content is also observed when $\mu_{\epsilon}=0^{\circ}$, but only near the peaks of the large wing shock. Figure 7 (c) quantifies the sensitivity observed in the pressure signatures, showing a large increase when $\mu_{\epsilon}=0.4^{\circ}$. A good compromise appears to be achieved with $\mu_{\epsilon}=0.2^{\circ}$, which is held fixed for the remainder of the analysis.

Stretching ratio is the next grid parameter examined. Figure 8 (a) shows the effect of the stretching ratio on the grid spacing in the space marching direction. For the purpose of this parameter study the aspect ratio was not fixed to 20, but instead allowed to grow as large as the prescribed stretching ratio evaluated to. This was very large even for the $S R=1.05$ case which resulted in an aspect ratio just below 100. The pressure signatures plotted in Figure 8 (b) and (d) show an increasing phase error in the pressure wave form with increasing stretching ratio. Even at a stretching ratio of $S R=1.05$ the phase error is still observed, illustrating that stretching ratio is a sensitive grid parameter for nonlinear wave propagation. Figure 8 (c) indicates that the sensitivity increases with increasing stretching ratio with a 10 percent reduction in the function evaluation with $S R=1.2$. Since the aspect ratio was not fixed in this study, a stretching ratio of $S R=1.05$ is chosen for this study.

Based on the large phase errors associated with large stretching ratios when the maximum aspect ratio is not bounded, a fixed stretching ratio of $S R=1.05$ with maximum aspect ratios of $A R=5,10,20$, and 40 are explored. Figure 9 (a) shows the effect of two different maximum aspect ratios on the space marching grid. In contrast to the large sensitivities observed with respect to stretching ratio, very small sensitivities are observed with respect to maximum aspect ratio (Figure 9 (b)-(d)). Almost no sensitivity is observed in Equation 14, and only a minor phase shift in the pressure wave form is observed at $A R=50$. Since a larger aspect ratio reduces the number of grid points in the space marching direction, a maximum aspect ratio of $A R=20$ is suggested from the analysis.

The streamwise resolution, $\Delta s$, of the space marching grid is controlled by the grid parameter $N_{\text {strm }}$. This parameter also controls the resolution in the space marching direction, in connection with stretching ratio and maximum aspect ratio (see Figure 10 (a)). In this study, $N_{\text {strm }}=155,309,617$, and 1233 are used to generate four space marching grids with increasing resolution on each $(\xi, \zeta)$ plane. This is equivalent to $\Delta s=1 / 2 \mathrm{~m}, 1 / 4 \mathrm{~m}, 1 / 8 \mathrm{~m}$, and $1 / 16 \mathrm{~m}$ (or $\left.\Delta s / L_{\text {ref }}=0.012,0.006,0.003,0.0015\right)$. Figure 10 (c) shows monotonic convergence of Equation 14 with increasing mesh resolution, which is desired but not guaranteed for this functional. Figure 10 (b) and (d), show that the pressure signatures are converging towards the fine CFD reference solution as the grid is refined, and the signatures generated using a $\Delta s=1 / 8 \mathrm{~m}$ and $\Delta s=1 / 16 \mathrm{~m}$ are nearly indistinguishable.

Sensitivity of the off-track pressure signature to variations in circumferential resolution $\Delta \theta$ are plotted 
in Figure 11. Four different circumferential spacings are considered, $\Delta \theta=1 / 2^{\circ}, 1^{\circ}, 2^{\circ}$, and $4^{\circ}$. Almost no variation in the pressure signature is observed with changes in circumferential spacing. Figure 11 (d) shows a zoomed in view of the pressure signature downstream of the wing shock, where very small variations in the pressure recovery are identified. This figure shows that as the circumferential spacing is decreased the space marching solution recovers the fine CFD reference solution in the recovery region.

With almost no sensitivity to circumferential spacing, one may question the importance of azimuthal dependence to nonlinear wave propagation. As a first step towards addressing this, a parameter study on the circumferential domain extent was performed. Space marching grids with four different circumferential domain extents were generated for $\theta \max =60^{\circ}, 90^{\circ}, 120^{\circ}$, and $180^{\circ}$ (see Figure 12 (a)). For grids with $\theta_{\max }<180^{\circ}$ extrapolation boundary conditions were used at the $\theta_{\max }$ computational boundary, while standard symmetry conditions were used at $\theta_{0}=0^{\circ}$ and $\theta \max =180^{\circ}$. Very large sensitivities are observed in the off-track pressure signature with respect to maximum circumferential domain extent. A pressure increasing effect is observed in Figure 12 (b) and (d) over the entire signature upstream of the wing shock, and anomalous behavior occurs in the recovery portion. This sensitivity is also observed in the functional in Figure $12(\mathrm{c})$ with more than 4 percent change in value when $\theta_{\max }=60^{\circ}$. This analysis suggests that either a full-cylinder or half-cylinder (for a symmetric aircraft) should be used for the space marching grid when the inner cylinder is located this close to the aircraft. The analysis also indicates that the entire half-body is required to capture the dipole effect from the wings, but the wave length of the dipole is large which is why the sensitivity to circumferential resolution is small. More detailed analysis of azimuthal dependence of nonlinear wave propagation is described in sub-Section III.B.

Based on the series of grid parameter studies, a Mach cone perturbation angle of $\mu_{\epsilon}=0.2^{\circ}$, stretching ratio of $S R=1.05$, maximum aspect ratio of $A R=20$, streamwise spacing of $\Delta s / L_{\text {ref }}=0.003$, circumferential spacing of $\Delta \theta=0^{\circ}$, and a maximum circumferential domain extend of $\theta_{\max }=180^{\circ}$ are sufficient for generating the space marching grid. Next, sensitivity to the solver parameters is explored.

\section{III.A.2. Solver Parameters}

It is well-known that consistent discretization of the metric terms is necessary in order to preserve freestream in generalized curvilinear coordinates. ${ }^{47}$ Using identical finite-difference operators for the metric terms and the convective flux derivatives leads to a consistent metric term discretization. ${ }^{48,49}$ For the space marching method, this implies that the HWCNS is used to discretize derivatives with respect to $\xi$ and $\eta$, while second-order (or first-order) backward differencing must be used in the $\zeta$ direction in order to preserve free-stream. This is illustrated in Figure 13 (a) and (b) where standard metric term evaluations are used in all three directions for sub-plot (a), while the consistent conservative metric method is used for subplot (b). The standard metric term evaluation leads to on $\mathcal{O}\left(10^{-5}\right)$ free-stream preservation errors. These errors are triggered when the grid starts stretching in the space marching direction, and are then triggered again when the grid abruptly stops stretching once the maximum aspect ratio limit is reached. In contrast, the conservative metric method results in $\mathcal{O}\left(10^{-13}\right)$ errors in free-stream preservation which are randomly distributed throughout the mesh. Results from applying the different metric term discretizations to the propagation of the near-field pressure signature are plotted in Figure 14 (a) and (b). Almost no difference can be identified when looking at the entire signature, zooming into the front portion of the signature, where $\Delta p / p_{\infty}=0$, we see that the standard metric method results in this same $\mathcal{O}\left(10^{-5}\right)$ error. Although this error may seem small, it will persist throughout the nonlinear wave propagation and inevitably corrupt the signatures whose amplitudes decay like $R^{1 / 2}$. The conservative metric method is obviously the preferred choice.

The fourth-order HWCNS with optimal third-order upwind biased left and right state interpolation (HWCNS4-OPT3) was chosen for the convective flux discretization in the $\xi$ and $\eta$ directions for all of the parameter studies performed thus far. This was mostly due to the superior nonlinear residual convergence properties of this discretization. It was pointed out in several of the grid parameter studies that spurious waves were observed for certain parameter values. This was partially attributed to the unlimited left and right state interpolation. In this parameter study, the effect of the choice in left and right state interpolation is assessed. Two additional interpolation options are tried; these include third-order WENO (HWCNS4WENO3) and fifth-order ZWENO (HWCNS-ZWENO5). In order to assess the different convective flux discretization options, the space marching solver was applied to the coarse $\left(\Delta s / L_{\text {ref }}=0.012\right)$ and medium $\left(\Delta s / L_{\text {ref }}=0.006\right)$ mesh resolutions from the streamwise spacing study, and compared to the space marching solution using the baseline parameters on the finest space marching grid $\left(\Delta s / L_{\text {ref }}=0.0015\right)$. Figure 15 
(a) and (b) show a comparison of the generated pressure signatures on the coarse grid. The unlimited interpolation option (HWCNS4-OPT3) contains some spurious wave content near the wing shock, while HWCNS4-WENO3 appears highly damped over this large feature. The HWCNS4-ZWENO5 option appears superior to the others, maintaining good amplitude levels through the wing shock while being devoid of clearly unphysical wave content. None of the interpolation options lead to good resolution of the wave train upstream of the wing shock at this mesh resolution. When applying these methods to the medium grid (Figure 15 (c) and (d)) a significant improvement in the resolution of the upstream wave train is observed for all of the methods. A significant reduction in the spurious wave content is produced by the unlimited HWCNS4-OPT3 method as the mesh is refined. The HWCNS4-ZWENO5 still shows the best resolution over the wing shock compared to HWCNS4-WENO3 and is on-par with HWCNS4-OPT3, while not containing any observable spurious wave content. The main negative attribute of using the HWCNS4-ZWENO5 option is the lack of nonlinear residual convergence. During the space marching sub-iteration procedure on a constant $\zeta$ plane, the ZWENO5 interpolation procedure produces limiter rattling, causing the residual convergence to stall. The effect of not deeply converging the nonlinear residuals is examined next.

A strict nonlinear residual tolerance of eight-orders of magnitude residual reduction was used for all of the grid sensitivity studies. It was observed in the study on interpolation options that the WENO and ZWENO options can lead to residual convergence stalling. When this occurs the space marching solver performs the maximum number of sub-iterations prescribed in the input file, 100 for the current study, then moves onto the next space marching station. Since the HWCNS4-OPT3 method is capable of achieving the tight residual tolerances, this method is applied with more relaxed convergence tolerances of $\epsilon_{\mathrm{conv}}=10^{-1}$, $10^{-2}, 10^{-4}$, and the strict tolerance of $10^{-8}$. The resulting pressure signatures are plotted in Figure 16 (a) and (b). A small phase error is observed for the most relaxed tolerance of $\epsilon_{\mathrm{conv}}=10^{-1}$, while no difference is observed between the signatures using the tighter convergence tolerances. This lack of sensitivity to residual convergence tolerance for $\epsilon_{\mathrm{conv}} \leq 10^{-2}$ is further demonstrated in the functional sensitivity plot (Figure 16 (c)). The average number of sub-iterations used during the space marching sweep for each residual convergence tolerance is plotted in Figure $16(\mathrm{~d})$. The sufficient convergence tolerance of $\epsilon_{\mathrm{conv}}=10^{-2}$ required between 5-6 sub-iterations. This test was then repeated with HWCNS4-ZWENO5, but instead of varying the residual convergence tolerance, the number of sub-iterations was varied from $n s u b=5,10,20$, and 40. Analogous to the sensitivity to residual convergence tolerance, insufficient number of sub-iterations results in a phase error in the pressure wave signature (Figure 17 (a) and (b)). Functional sensitivity (Figure 17 (c)) suggests that 10 sub-iterations is sufficient for the HWCNS4-ZWENO5. Efficiency of the two interpolation options is compared in Figure 17 (d) where the wall-clock time is plotted as a function of average number of sub-iterations for both the HWCNS4-OPT3 and HWCNS4-ZWENO5 methods. The HWCNS4-OPT3 required 84 seconds for the space marching solve using $\epsilon_{\mathrm{Conv}}=10^{-2}$, while the HWCNS4ZWENO5 required 138 seconds using $n s u b=10$. Since the HWCNS4-ZWENO5 method has superior wave amplitude resolution properties and is devoid of spurious wave content, it is the recommended convective flux discretization option based on this study.

\section{III.B. Azimuthal Dependence of Nonlinear Wave Propagation}

After completing the grid and solver sensitivity analyses for the JWB and examining the "on-track" $(\theta=0$ degrees) and "off-track" ( $\theta=50$ degrees) pressure signatures, an interesting phenomenon was observed in the propagated signature generated from the aft end of the vehicle, i.e. the shock/expansion generated from the wing. It is well known that interfacing CFD directly to the far-field wave propagation code at a radial location that is "too close" to the aircraft can lead to erroneous ground level sound pressures. ${ }^{11}$ The main driver requiring an extended radial CFD domain, or in this case the introduction of the space marching domain, is to capture the non-negligible diffraction effects caused by azimuthal variations generated by the aircraft. This azimuthal dependence can persist for several body lengths in the radial direction. In fact, George ${ }^{15}$ showed that the wing of a supersonic aircraft can be manipulated to generate a dipole, which can redistribute the on-track pressure to an off-track azimuth. Since the cylindrical acoustic wave at an off-track azimuth will take longer to reach the ground (at steady cruise conditions and a no-wind atmosphere), the overall noise footprint can be reduced. It is this redistribution of the on-track pressure that is observed in the aft portion of the pressure signature for the JWB configuration.

The explanation of this phenomenon is split into to two complementary parts; near-field to mid-field and mid-field to the ground. Figure 18 plots a diagram of the flow-field to illustrate the influence of the azimuthal velocity. Contour lines of azimuthal velocity are plotted at streamwise stations of $x / L_{r e f}=2,4,6$, and 8 . 
At the same time, pressure contours are plotted at several distances below the aircraft, $z / L_{r e f}=-2,-4$, -6 , and -8 . The inset image shows a zoomed in view of the JWB with contour lines of azimuthal velocity shown on-top of a pressure contour at a constant streamwise location. The image clearly shows the dipole shape formed by the azimuthal velocity contours as they cut through the pressure contour slices. Details of this interaction are described next.

\section{III.B.1. Near-Field to Mid-Field}

To begin the near-field to mid-field analysis, Figures 19 - 22, show a flood plot of pressure for each altitude below the vehicle as depicted in Figure 18. In addition to the pressure contour flood, 12 equally spaced contour lines of the magnitude of azimuthal velocity, from $0.01 \mathrm{~m} / \mathrm{s} \leq\left|U_{\theta}\right| \leq 10.0 \mathrm{~m} / \mathrm{s}$, are plotted as black lines on-top of the flood. The concentration of azimuthal velocity contour lines appear to surround the aft shock/expansion generated by the wing. In order to assess the effect of this concentration of azimuthal velocity on the nonlinear wave propagation, the on-track pressure signature is extracted at 8 different radial distances below the aircraft, $r / L_{\mathrm{ref}}=1,2, \cdots, 8$ (Figure 23) and plotted simultaneously.

The pressure signatures for each $r / L_{\text {ref }}$ are plotted in Figure 24 (a) and (b). To facilitate the comparison of the pressure signatures, the non-dimensional variable $(x-\beta r) / L_{\text {ref }}$ is used for the x-axis, which accounts for the spatial translation of the signatures as a function of radial extraction distance by using the PrandtlGlauert transformation. In addition, the amplitudes of the pressure signature are rescaled by $R^{1 / 2}$, which is the decay rate for a cylindrical acoustic wave. If azimuthal effects are completely negligible, then there should be little to no difference in local pressure amplitude, with wave forms simply moving upstream (or downstream) if it is forming a shock (or expansion). This appears to be the case for most of the $\mathrm{x}$-domain, but Figure 24 (b) clearly shows that the strong wing shock (and expansion) is decaying at a much faster rate than $R^{1 / 2}$. The faster decay rate indicates that this wave is not obeying cylindrical acoustic wave theory, which assumes no azimuthal dependence. The location of this quickly decaying wave form is directly correlated to the area where the concentration of azimuthal velocity is high, as already shown in Figures 19 22. This implies that the nonlinear wave propagation is highly dependent on azimuthal effects, at least locally near the aft-shock from the wing. Moreover, Figure 25 shows a polar contour plot of the maximum magnitude of azimuthal velocity, $\max _{x}\left|U_{\theta}(r, \theta)\right|$, illustrating that the dipole effect from the lifting wing is non-vanishing even out to 10 body lengths away from the aircraft. The effect of this azimuthal dependence is further demonstrated by propagating each of the signatures to the ground, which is discussed in the next sub-section.

\section{III.B.2. Mid-Field to Ground}

To assess the effect of azimuthal dependence on the ground level noise, each of the 8 on-track pressure signatures, located at different radial distances below the aircraft, were propagated to the ground using the far-field sonic boom propagation code sBOOM. ${ }^{6}$ Figure 26 plots the overpressure ground signature as a function of time for each of the starting mid-field extraction locations. A very large difference in wave form shape is observed between the $r / L_{\text {ref }}=1$ extraction location and all other radial extraction locations. This is expected, since it is well understood that one body length away from the aircraft is too close to interface with sBOOM. It is also evident that the difference in ground signatures decreases with increasing radial extraction location. This is also expected based on physical reasoning that the azimuthal effect from the lifting body will eventually decay to a negligible value. To be more precise, Figure 27 plots the perceived loudness of the ground signature as a function of radial extraction distance from below the aircraft. This quantity represents the relative annoyance the aircraft would cause to a human on the ground as the plane flies over-head. The plot indicates that a radial extent of $r / L_{\mathrm{ref}} \geq 4$ is necessary for the loudness metric to become insensitive to radial extraction distance for the JWB. This outcome has several consequences:

- First it confirms the conclusion from the near-field to mid-field analysis, i.e. that azimuthal dependence of nonlinear wave propagation is not negligible and can persists to more than 4 body lengths away from the aircraft.

- This also demonstrates that assessing the sensitivity to radial extraction distance is important (up to $0.6 \mathrm{~dB}$ for the JWB), and should be performed for every configuration and every azimuth.

- Lastly it verifies that the space marching procedure is able to accurately propagate weak non-linear waves up to (and passed) 8 body lengths without introducing excessive artificial dissipation. This 
conclusion comes from the observation that the perceived noise metric predicted by the space marching method becomes independent with respect to radial extraction distances beyond 4 body lengths. If excessive artificial dissipation is added during the propagation process, the ground level noise metric would not converge to a fixed value.

\section{III.C. Low Boom Aircraft Wind Tunnel Model: Lockheed Martin 1021}

The best practices for the space marching method, established through the grid and solver sensitivity studies, along with the insight gained through the azimuthal dependence analysis performed on the JWB, are now applied to the Lockheed Martin Phase I low sonic boom model from the First AIAA Sonic Boom Prediction Workshop (SBPW1). The LM-1021, was designed to achieve low-boom on-track signatures and reduced off-track overpressures up to 20 degrees. The reference length of the LM 1021 is 22.365 inches and represents a 0.008 percent $(1: 125)$ model of the flight-scale aircraft. A blade strut is used to hold the model in place and is swept greater than the Mach angle to minimize interference with the measured pressure signatures. Figure 28 shows a three-view of the configuration. Note that one of the nacelles is located behind the blade strut between the v-shaped tail. The simulations were performed at Mach 1.6 with a Reynolds number of 4.36 million and an angle of attack of 2.1 degrees. Experimental data, obtained from Cliff et. al, ${ }^{3}$ for on-track measurements at this angle of attack are used for validation. The near-field CFD was performed during SBPW1 using a structured overset grid system and the LAVA CFD solver, details can be found in Housman et. al. ${ }^{2}$

\section{III.C.1. Space Marching Computational Grid}

Inputs for the space marching grid generation procedure were taken directly from the grid sensitivity studies completed for the JWB. These inputs include a Mach cone perturbation angle of $\mu_{\epsilon}=0.2^{\circ}$, a stretching ratio of 1.05 with a maximum aspect ratio of 20 , a streamwise grid spacing of $\Delta s / L_{r e f}=0.003$, a circumferential spacing of $\Delta \theta=1^{\circ}$, a maximum circumferential extent of $\theta \max =180^{\circ}$, and maximum radial extent of 10 body lengths. This resulted in a $351 \times 181 \times 564$ space marching grid (35.8 million grid points) which required 4.2 seconds to generate. Figure 29 plots the space marching grid (in magenta) overlaying the CFD mesh (gray) generated for the workshop on the symmetry plane. The radial extent of the space marching grid is considerably larger than the CFD grid used for SBPW1.

\section{III.C.2. Wind Tunnel Comparison}

The HWCNS4-ZWENO5 convective flux discretization with 20 sub-iterations was used to propagate the near-field CFD solution from $r / L_{\text {ref }}=0.14$ to $r / L_{\text {ref }}=10$. The computation required 106 seconds of wallclock time using 80 OpenMP threads on a single workstation. In comparison, the CFD simulation used a second order accurate Roe convective flux discretization along with the Spalart-Allmaras turbulence model, and required 1 hour and 30 minutes using 9 Ivy Bridge nodes (180 cores) at the time of the workshop in 2014. The solution is extracted at four on-track radial distances from the LM-1021 model for comparison with available experimental data. These locations include $r / L_{r e f}=0.93,1.4,1.88$, and 3.11 (Figure 30). Pressure signatures generated using the space marching method are compared to both existing CFD data, and wind tunnel measurements for each radial distance in Figures 31 (a) - (d). The space marching solution appears to predict larger amplitude and sharper pressure wave forms than the CFD. This is due to the increased accuracy of the HWCNS4-ZWENO5 discretization used in the space marching method compared to the standard second-order accurate Roe discretization used in the CFD simulation. When comparing to the experimental measurements, the pressure signatures generated using space marching method compares well overall, but again shows much sharper features and contains larger amplitudes than the experimental data. The original second-order accurate CFD solution shows similar differences with the experimental measurements. This is caused by the signature averaging procedure used to remove tunnel noise from the wind tunnel measurements. This noise can be as large as the pressure amplitudes generated by the low boom models themselves. In order to account for the noise in the tunnel, the signatures are measured with with the model located at several axial locations relative to the pressure rail. The signatures are then lined up using the nose shock as a reference and averaged to produce the final signature. This has the effect of smoothing out shocks and reducing the amplitude of pressure peaks. A detailed discussion of the wind tunnel measurement procedure and the smoothing effects of the averaging procedure are provided in Cliff 
et. $a l .{ }^{3}$ Overall, the space marching solution appears as good as the second-order accurate CFD solution results when comparing to the experimental measurements, validating the space marching method.

\section{Summary}

A high-order accurate space marching method was developed for efficient near-field to mid-field sonic boom propagation. The method solves the Euler equations on an automatically generated structured orthogonal Mach-cone aligned curvilinear grid using a high-order accurate finite-difference discretization and a single-sweep nonlinear space marching method. Mesh converged results propagated to 10 body lengths from the aircraft are computed in approximately 3 minutes on a single workstation. This is in comparison to traditional CFD simulations which require several hours using 100's or 1000's of cores.

A three-stage process for computing ground level noise from an aircraft is evaluated as a replacement of the current two-stage approach. This includes using near-field CFD on a significantly reduced radial domain extending just pass the semi-span of the wing, then propagating the near-field solution to the mid-field using the space marching procedure, and finally propagating the mid-field solution to the ground using a far-field propagation code. The benefit of this approach for generating a large database for a supersonic aircraft for flight planning is now discussed.

Considering angle of attack, side-slip angle, free-stream conditions, control deflections, power settings, etc., this may require $\mathcal{O}\left(10^{3}-10^{4}\right)$ simulations. Since sBOOM only requires $\mathcal{O}\left(10^{1}\right)$ seconds to run on a workstation, and can be run for each configuration of the database independently, the far-field propagation portion of the procedure is not a bottleneck. If the current two-stage approach is utilized, the results from the JWB analysis suggest that the radial extent of the CFD domain must be at least 5 or 6 body lengths from the aircraft in order to perform the radial extraction sensitivity analysis. Currently in the LBFD program, extraction of the pressure signature occurs at a radial distance of only 3 body lengths. This implies that the radial extent of the CFD domain must effectively double in order to properly assess the influence of the radial interface location between CFD and sBOOM (or any other far-field propagation code). At best, the CFD mesh resolution requirements for this extended region will remain fixed, i.e. no additional grid stretching and a fixed aspect ratio will be required to properly resolve the pressure waves (without effects from artificial dissipation). This will lead to approximately 20-40 percent more grid points and at least that amount of additional computational resources. For example, the original CFD simulation performed for the SBPW2 required 6 hours using 16 Ivy Bridges nodes (320 cores) and had a radial extent of just under 7 body lengths.

Alternatively, if the three-stage procedure outlined in the current work is utilized, then the CFD domain extent can be significantly reduced leading to a 20-40 percent reduction in grid points along with a similar reduction in computational resources. For the JWB near-field CFD simulation, the wall-clock time was reduced to 4 hours using only 8 Ivy Bridges nodes (160 cores) and had a radial extent of just over 0.3 body lengths. This is a 66 percent reduction in the computational resources compared to the SBPW2 simulation. The timing for the space marching portion of the process for the JWB configuration, on a single workstation $(2 \times$ Xeon Gold 6152 CPUs) was 13.6 seconds for the automatically generated space marching grid (116.4 million grid points and radial extent of 10 body lengths), 7.5 seconds to interpolate the 223 thousand fringe points from the CFD solution using 40 MPI ranks, and 165 seconds for the space marching solution using the HWCNS4-ZWENO5 with 10 sub-iterations and 80 OpenMP threads. This is a total of 3 minutes and 6 seconds, and analogous to sBOOM, can be run for each configuration of the database independently (but only requires one simulation for all of the azimuthal signatures). The computational resources of the space marching method are insignificant compared to the cost of the CFD simulation, which implies that any reduction in computational expense by reducing the radial extent of the CFD domain directly relates to an overall reduction in cost for generating the database. Based on the JWB results this leads to more than a 60 percent reduction in computational resources. At the same time, ensuring that the radial distance of the interface region between the mid-field and far-field propagation codes is far enough away for accurate (independent) ground noise level predictions.

Detailed analysis of the azimuthal dependence of weak nonlinear wave propagation was performed for the JAXA wing body. Rapid decay of the pressure amplitude of the shock/expansion generated by the wing was directly correlated to the presence of azimuthal velocity. The azimuthal velocity is generated by a dipole effect caused from the lift of the wing, and was shown to be non-zero even out to 10 body lengths from the aircraft. The effect of the azimuthal velocity on ground level noise was demonstrated by propagating on-track 
pressure signatures from a series of radial distance from below the aircraft using the far-field propagation code sBOOM. It was demonstrated that a radial distance of greater than or equal to 4 body lengths was required before the mid-field azimuthal velocity effects were negligible.

Evaluation of the sensitivity to grid and solver parameters was studied through a series of parameter studies. A set of baseline parameters was selected from using the sensitivity analysis and applied to the Lockheed Martin 1021 from the First AIAA Sonic Boom Prediction Workshop. A 35.8 million grid point space marching grid was automatically generated in 4.2 seconds, and the single-sweep space marching method propagated the near-field solution to 10 body lengths from the wind tunnel test article in 106 seconds on a single workstation. Good comparisons with both existing CFD and wind tunnel measurements were obtained, and the accurate wave resolution properties of the high-order space marching method were demonstrated.

Given the high accuracy and low computational cost of the fully three-dimensional space marching method developed for near-field to mid-field propagation, we are examining ways to extend the methodology to propagate directly to the ground. Fully three-dimensional simulations of the Euler equations, including atmospheric effects, for sonic boom propagation to the ground have been reported by Yamashita and Suzuki. ${ }^{53,54}$ In their approach, hydrostatic effects of gravity are accounted for by solving for the nonlinear perturbation about a known atmospheric condition. This is similar to the approach used by Buning and Steger ${ }^{55}$ for handling free-stream preservation on curvilinear grids using flux vector splitting schemes. We propose to follow this path, but utilize the highly efficient and accurate space marching solution procedure instead of the traditional CFD approach used in Yamashita and Suzuki. ${ }^{53,54}$ This should keep the computational costs reasonable, while allowing the primary boom carpet to be captured in a single simulation, including all relevant azimuthal effects and atmospheric effects.

\section{Acknowledgements}

This work was partially supported by the NASA's ARMD Commercial Supersonic Technologies (CST) project. The authors would like to thank the members of the AIAA Sonic Boom Prediction Workshop organizing committee, as well as Marian Nemec and William Chan of NASA Ames Research Center for valuable discussions. Computer time has been provided by NASA's Advanced Supercomputing (NAS) facility at NASA Ames Research Center.

\section{References}

\footnotetext{
${ }^{1}$ National Aeronautics and Space Administration, "Low-Boom Flight Demonstration Overview," https://www.nasa.gov/mission_pages/lowboom/overview.

${ }^{2}$ Housman, J., Sozer, E., Moini-Yekta, S., and Kiris, C., "LAVA Simulations for the AIAA Sonic Boom Prediction Workshop," 32nd AIAA Applied Aerodynamics Conference, Atlanta, GA, June 2014, AIAA-2014-2008.

${ }^{3}$ Cliff, S., Durston, D., Elmiligui, A., Jensen, J., and Chan, W., "Computational and Experimental Assessment of Models for the First AIAA Sonic Boom Prediction Workshop," 52nd Aerospace Sciences Meeting, National Harbor, Maryland, January 2014, AIAA-2014-0560.

${ }^{4}$ Aftosmis, M. and Nemec, M., "Cart3D Simulations for the First AIAA Sonic Boom Prediction Workshop," 52nd Aerospace Sciences Meeting, National Harbor, Maryland, January 2014, AIAA-2014-0558.

${ }^{5}$ Anderson, G., Aftosmis, M., and Nemec, M., "Cart3D Simulations for the Second AIAA Sonic Boom Prediction Workshop," Journal of Aircraft, August 2018, in print.

${ }^{6}$ Rallabhandi, S., "Advanced Sonic Boom Prediction Using the Augmented Burgers Equation," Journal of Aircraft, Vol. 48, No. 4, July-August 2011.

${ }^{7}$ Rallabhandi, S. and Loubeau, A., "Summary of Propagation Cases of the Second AIAA Sonic Boom Prediction Workshop," 35th AIAA Applied Aerodynamics Conference, Denver, Colorado, June 2017, AIAA-2017-3257.

${ }^{8}$ Rallabhandi, S., "Sonic Boom Prediction and Mitigation using Three-Dimensional Earth Effects," AIAA Applied Aerodynamics Conference, Atlanta, Georgia, June 2018, AIAA-2018-2848.

${ }^{9}$ Ferri, A., Ting, L., and Lo, R., "Nonlinear Sonic Boom Propagation Including the Asymmetric Effects," AIAA J., Vol. 15, No. 5, 1977, pp. 653-658.

${ }^{10}$ Darden, C., "An Analysis of Shock Coalescence Including Three-Dimensional Effects with Application to Sonic Boom Extrapolation," NASA Technical Paper 2214, January 1984, NASA-TP-2214.

${ }^{11}$ Page, J. and Plotkin, K., "An Efficient Method for Incorporating Computational Fluid Dynamics into Sonic Boom Prediction," 9th AIAA Applied Aerodynamics Conference, Baltimore, MD, September 1991, AIAA-1991-3275.

${ }^{12}$ Plotkin, K. and Page, J., "Extrapolation of Sonic Boom Signatures from CFD solutions," 40th Aerospace Sciences Meeting, Reno, Nevada, January 2002, AIAA-2002-0922.

${ }^{13}$ Rallabhandi, S. and Mavris, D., "New Computational Procedure for Incorporating Computational Fluid Dynamics into Sonic Boom Prediction," Journal of Aircraft, Vol. 44, No. 6, November-December 2006.

${ }^{14}$ Saito, Y., Ukai, T., Miyakoshi, K., Ohtani, K., and Obayashi, S., "Sonic Boom Estimation using the Multipole Method for Free-Flight Experiments," 52nd Aerospace Sciences Meeting, National Harbor, Maryland, January 2014, AIAA-2014-0368.
} 
${ }^{15}$ George, A., "Reduction of Sonic Boom by Azimuthal Redistribution of Overpressure," AIAA J., Vol. 15, No. 5, 1977, pp. 653-658.

${ }^{16}$ Whitham, G., "On the Propagation of Weak Shock Waves," Journal of Fluid Mechanics, Vol. 290, 1956.

${ }^{17}$ Kandil, O., Yang, Z., and Bobbitt, P., "Prediction of Sonic Boom Signatures using Euler-Full Potential CFD with Grid Adaptation and Shock Fitting," 54th Aerospace Sciences Meeting, San Diego, California, January 2016, AIAA-2016-2037.

${ }^{18}$ Kandil, O., Ozcer, I., Zheng, X., and Bobbitt, P., "Comparison of Full-Potential Propagation-Code Computations with the F-5E Shaped Sonic Boom Experiment Program," 54th Aerospace Sciences Meeting, San Diego, California, January 2016, AIAA-2016-2037.

${ }^{19}$ Cheung, S., Edwards, T., and Lawrence, S., "Application of CFD to Sonic Boom Near and Mid-Field Prediction," $13 t h$ AIAA Aeroacoustics Conference, Tallahassee, FL, October 1990, AIAA-1990-3999.

${ }^{20}$ Siclari, M. and Darden, C., "An Euler Code Prediction of Near to Mid-Field Sonic Boom Pressure Signatures," 13th AIAA Aeroacoustics Conference, Tallahassee, FL, October 1990, AIAA-1990-4000.

${ }^{21}$ Shen, H. and Lazzara, D., "A Space Marching Method for Sonic Boom Near Field Predictions," 54th Aerospace Sciences Meeting, San Diego, California, January 2016, AIAA-2016-2037.

${ }^{22}$ Rubin, S. and Lin, T., "A Numerical Method for Three-Dimensional Viscous Flow: Application to the Hypersonic Leading Edge," Journal of Computational Physics, Vol. 9, No. 2, 1972, pp. 339-364.

${ }^{23}$ Vigneron, Y., Rakich, J., and Tannehill, J., "Calculation of Supersonic Viscous Flow over Delta Wings with Sharp Subsonic Leading Edges," 11th AIAA Fluid and Plasma Dynamics Conference, Seattle, Washington, July 1978, AIAA-19781137.

${ }^{24}$ Schiff, L. and Steger, J., "Numerical Simulation of Steady Supersonic Viscous Flow," 17th Aerospace Sciences Meeting, New Orleans, La., January 1979, AIAA-1979-0130.

${ }^{25}$ Chakravarthy, S. and Szema, K., "Euler Solver for Three-Dimensional Supersonic Flows with Subsonic Pockets," 18th Fluid Dynamics and Plasma Dynamics and Lasers Conference, Cincinnati, OH, 1985, AIAA-1985-1703.

${ }^{26}$ Ota, D., Chakravarthy, S., and Darling, J., "An Equilibrium Air Navier-Stokes Code for Hypersonic Flows," 26th AIAA Aerospace Sciences Meeting, Reno, Nevada, 1988, AIAA-1988-0419.

${ }^{27}$ Lawrence, S., Tannehill, J., and Chausee, D., "Upwind Algorithm for the Parabolized Navier-Stokes Equations," AIAA Journal, Vol. 27, No. 9, 1989, pp. 1175-1183.

${ }^{28}$ Shaw, S. and Qin, N., "A Matrix-Free Preconditioned Krylov-Subspace Method for the PNS Equations," 36th AIAA Aerospace Sciences Meeting, Reno, NV, 1998, AIAA-1998-0111.

${ }^{29}$ Qin, N., Ludlow, D., Zhong, B., Shaw, S., and Birch, T., "Multigrid Acceleration of a Preconditioned GMRES Implicit PNS Solver," 37th AIAA Aerospace Sciences Meeting, Reno, NV, 1999, AIAA-1999-0779.

${ }^{30}$ Birch, T., Prince, S., Simpson, G., and Ludlow, D., "IMPNS: A Space-Marching Solver for Predicting the Aerodynamic Characteristics of High-Speed Missiles," AIAA Atmospheric Flight Mechanics Conference, Monterey, California, August 2002, AIAA-2002-4512.

${ }^{31} \mathrm{de}$ Feo, D., Qin, N., and Birch, T., "A MUSCL and WENO-PNS Approach for Vortex Dominated Flowfields," 48th AIAA Aerospace Sciences Meeting, Orlando, FL, January 2010, AIAA-2010-0503.

${ }^{32}$ Kiris, C., Housman, J., Barad, M., Brehm, C., Sozer, E., and Moini-Yekta, S., "Computational Framework for Launch, Ascent, and Vehicle Aerodynamics (LAVA)," Aerospace Science and Technology, Vol. 55, August 2016, pp. 189-219.

${ }^{33}$ Vinokur, M., "Conservation Equations of Gasdynamics in Curvilinear Coordinate Systems," Journal of Computational Physics, Vol. 14, 1974, pp. 105-125.

${ }^{34}$ Choi, S., Alonso, J., and Ven der Weide, E., "Numerical and Mesh Resolution Requirements for Accurate Sonic Boom Prediction," Journal of Aircraft, Vol. 46, No. 4, July-August 2009.

${ }^{35}$ Park, M., Campbell, R., Elmiligui, A., Cliff, S., and Nayani, S., "Specialized CFD Grid Generation Methods for Near-Field Sonic Boom Prediction," 52nd AIA A Aerospace Sciences Meeting, National Harbor, Maryland, January 2014, AIAA-2014-0368.

${ }^{36}$ Deng, X., Mao, M., Jiang, Y., and Liu, H., "New High-Order Hybrid Cell-Edge and Cell-Node Weighted Compact Nonlinear Schemes," 20th AIAA Computational Fluid Dynamics Conference, Honolulu, Hawaii, June 2011, AIAA-2011-3857.

${ }^{37}$ Nonomura, T. and Fujii, K., "Robust explicit formulation of weighted compact nonlinear scheme," Computers 83 Fluids, Vol. 85, 2013, pp. 8-18.

${ }^{38}$ Housman, J. and Kiris, C., "Slat Noise Predictions using Higher-Order Finite-Difference Methods on Overset Grids," 22nd AIAA/CEAS Aeroacoustic Conference, Lyon, France, May 30-31 2016, AIAA-2016-2963.

${ }^{39}$ Housman, J., Kiris, C., and Hafez, M., "Preconditioned methods for simulations of low speed compressible flows," Computes and Fluids, Vol. 38, No. 7, August 2009, pp. 1411-1423.

${ }^{40}$ Housman, J., Kiris, C., and Hafez, M., "Time-Derivative Preconditioning Methods for Multicomponent Flows - Part I: Riemann Problems," Journal of Applied Mechanics, Vol. 76, No. 2, February 2009.

${ }^{41}$ Housman, J., Kiris, C., and Hafez, M., "Time-Derivative Preconditioning Methods for Multicomponent Flows - Part II: Two-Dimensional Applications," Journal of Applied Mechanics, Vol. 76, No. 3, March 2009.

${ }^{42}$ Shu, C.-W., "High Order Weighted Essentially Nonoscillatory Schemes for Convection Dominated Problems," SIAM Review, Vol. 51, No. 1, 2009, pp. 92-126.

${ }^{43}$ Borges, R., Carmona, M., Costa, B., and Don, W., "An improved weighted essentially non-oscillatory scheme for hyperbolic conservation laws," Journal of Computational Physics, Vol. 227, No. 6, 2008, pp. 3191-3211.

${ }^{44}$ Thornber, B., Mosedale, A., Drikakis, D., Youngs, D., and Williams, R. J., "An improved reconstruction method for compressible flow with low Mach number features," Theoretical and Computational Fluid Dynamics, Vol. 26, 2012, pp. 523-550.

${ }^{45}$ Housman, J., Stich, G., and Kiris, C., "Jet Noise Prediction using Hybrid RANS/LES with Structured Overset Grids," 23rd AIAA/CEAS Aeracoustics Conference, 2017, AIAA-2017-3213.

${ }^{46}$ Stich, G., Housman, J., Kocheemoolayil, J., Barad, M., and Kiris, C., "Application of Lattice Boltzmann and NavierStokes Methods to NASA's Wall Mounted Hump," 2018 Fluid Dynamics Conference, AIAA AVIATION Forum, 2018, AIAA2018-3855.

16 of 34 
${ }^{47}$ Thomas, P. and Lombard, C., "Geometric Conservation Law and Its Application to Flow Computations on Moving Grids," AIAA Journal, Vol. 17, No. 10, 1979, pp. 1030-1037.

${ }^{48}$ Vinokur, M. and Yee, H., "Extension of Efficient Low Dissipation High Order Schemes for 3-D Curvilinear Moving Grids," Frontiers of Computational Fluid Dynamics, World Scientific, 2002, pp. 129-163, edited by D. A. Caughey and M. M. Hafez.

${ }^{49}$ Deng, X., Mao, M., Tu, G., Liu, H., and Zhang, H., "Geometric conservation law and applications to high-order finite difference schemes with stationary grids," Journal of Computational Physics, Vol. 230, 2011, pp. 1100-1115.

${ }^{50}$ Housman, J. and Kiris, C., "Numerical Simulations of Shock/Plume Interaction using Structured Overset Grids," 33rd AIAA Applied Aerodynamics Conference, Dallas, TX, June 2015, AIAA-2015-2262.

${ }^{51}$ Pointwise, "Grid Generation Software," https://https://www.pointwise.com.

${ }^{52}$ Chan, W., "Developments in Strategies and Software Tools for Overset Structured Grid Generation and Connectivity," 20th AIAA Computational Fluid Dynamics Conference, Honolulu, Hawaii, June 2011, AIAA-2011-3051.

${ }^{53}$ Yamashita, R. and Suzuki, K., "Full-Field Sonic Boom Simulation in Stratified Atmosphere," AIAA Journal, Vol. 54, No. 10, Oct 2016.

${ }^{54}$ Yamashita, R. and Suzuki, K., "Rise Time Prediction of Sonic Boom by Full-Field Simulation," Journal of Aircraft, Vol. 55, No. 3, May-June 2018.

${ }^{55}$ Buning, P. and Steger, J., "Solution of the Two-Dimensional Euler Equations with Generalized Coordinate Transformation Using Flux Vector Splitting," AIAA/ASME 3rd Joint Thermophysics, Fluids, Plasma, and Heat Transfer Conference, St. Louis, Missouri, June 1982, AIAA-1982-0971. 


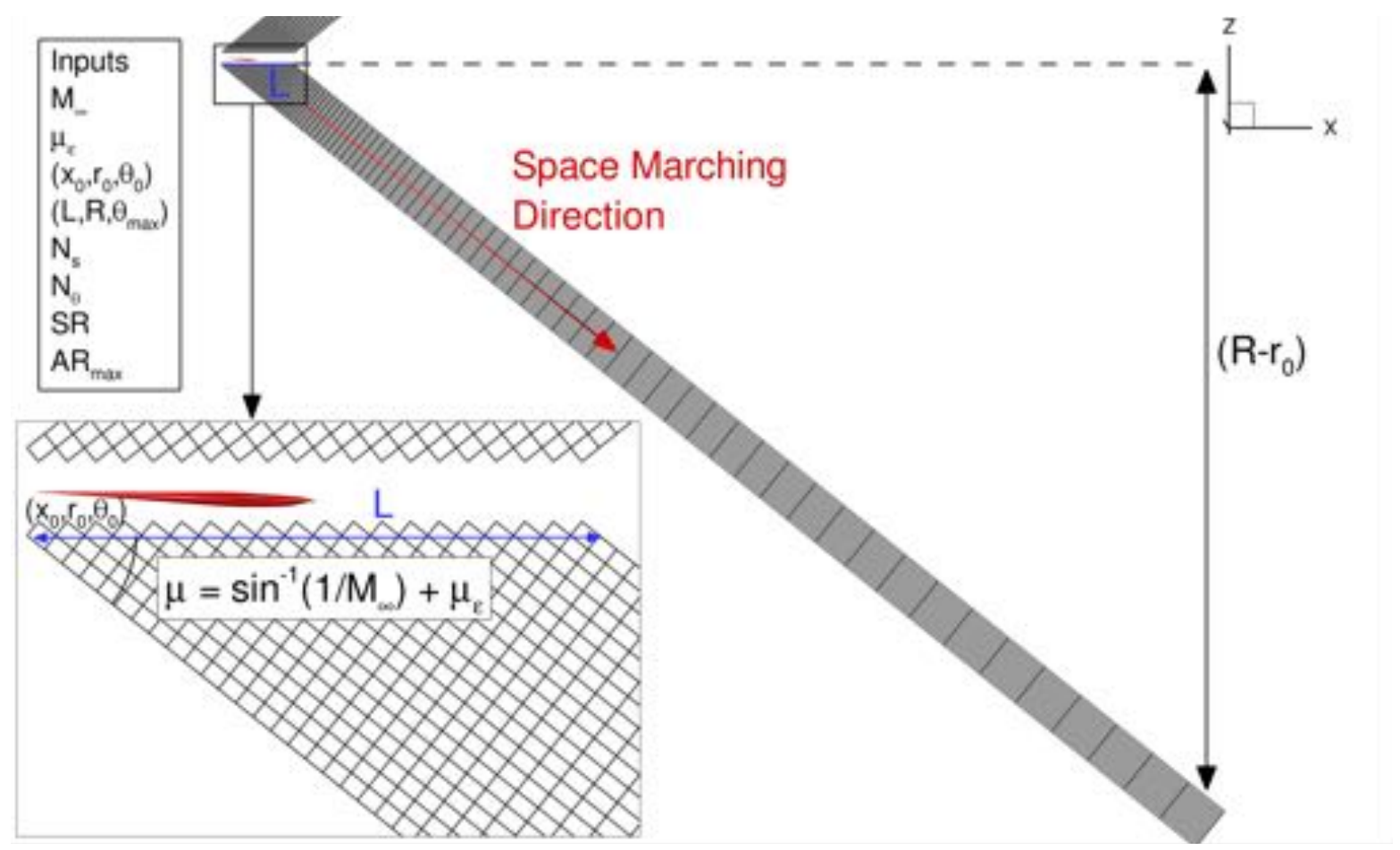

(a)

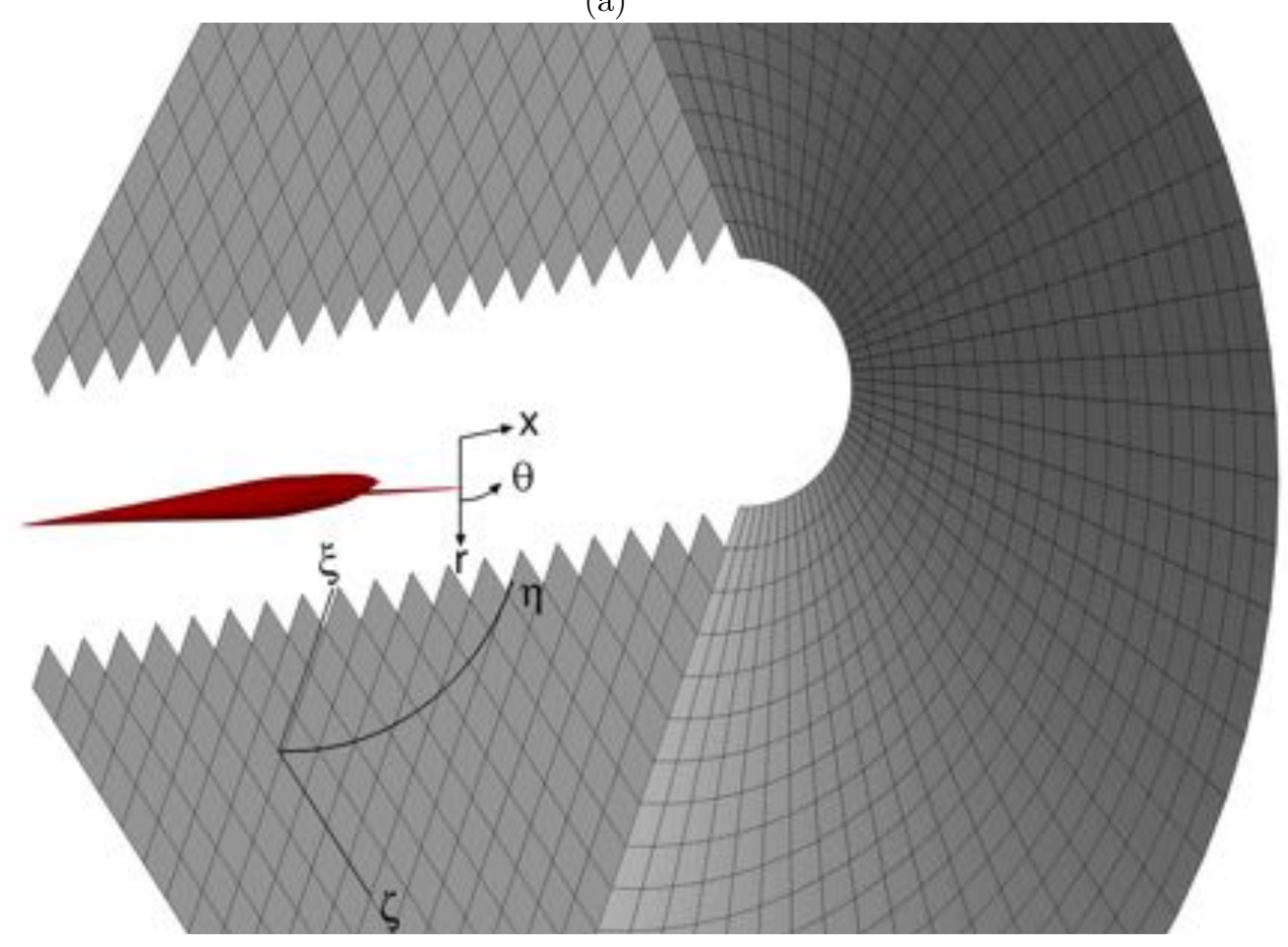

(b)

Figure 1. (a) Schematic of the structured curvilinear Mach-cone aligned cylindrical space marching grid including inputs for the grid generation program. The inputs include: $\mathbf{M}_{\infty}$ the free-stream Mach number; $\mu_{\epsilon}$ the Mach-cone perturbation angle, $\left(x_{0}, r_{0}, \theta_{0}\right)$ the starting axial, radial, and circumferential locations of the inner cylinder suface; $\left(\mathbf{L}, \mathbf{R}, \theta_{\max }\right)$ the axial length, radial extent, and circumferential extent of the mesh; $\mathbf{N}_{\mathbf{S}}$ and $\mathbf{N}_{\theta}$ the number of points in the streamwise axial and circumferential directions; SR and ARmax the stretching ratio and maximum aspect ratio in the space marching direction. (b) Iso-parametric view of the space marching grid showing the symmetry plane and a constant $\zeta$ slice in the space marching direction. The curvilinear coordinate system, $(\xi, \eta, \zeta)$, and cylindrical coordinate system, $(\mathbf{x}, \mathbf{r}, \theta)$, are also defined. 


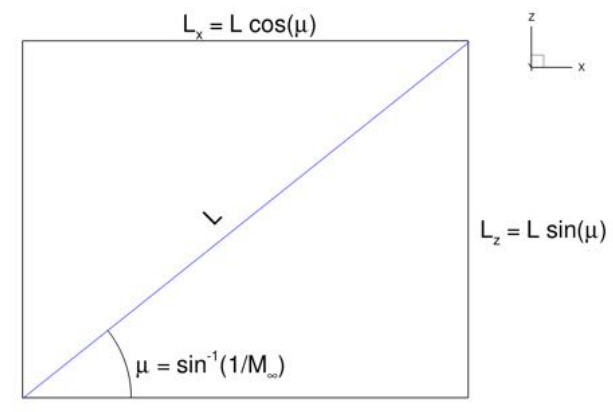

(a)

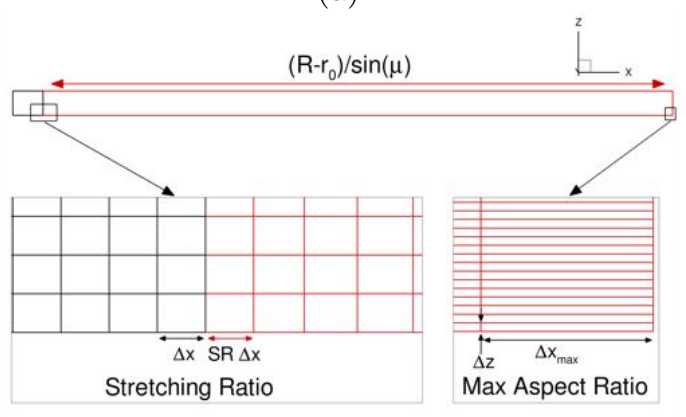

(c)

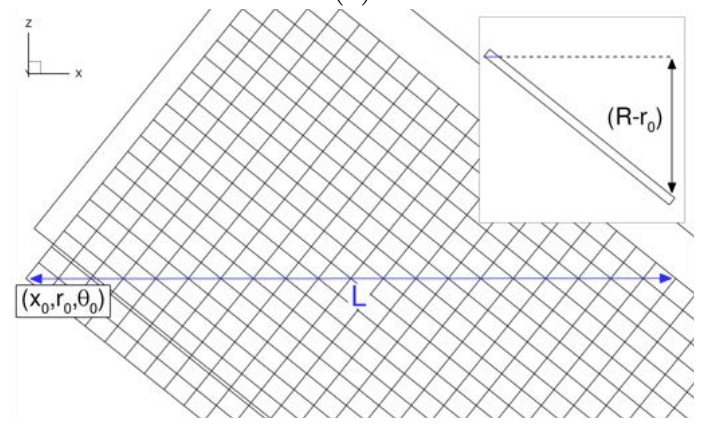

(e)

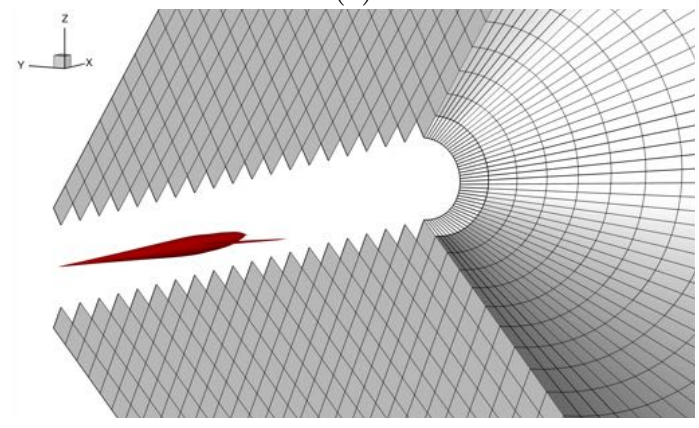

(g)

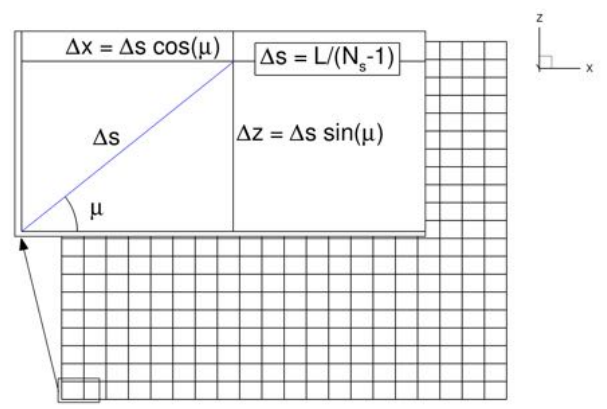

(b)

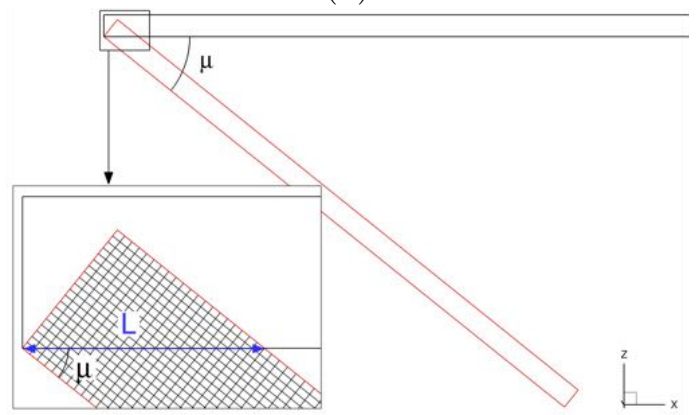

(d)

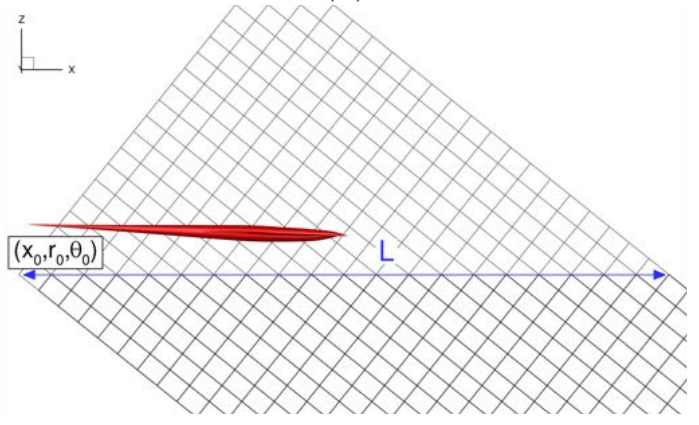

(f)

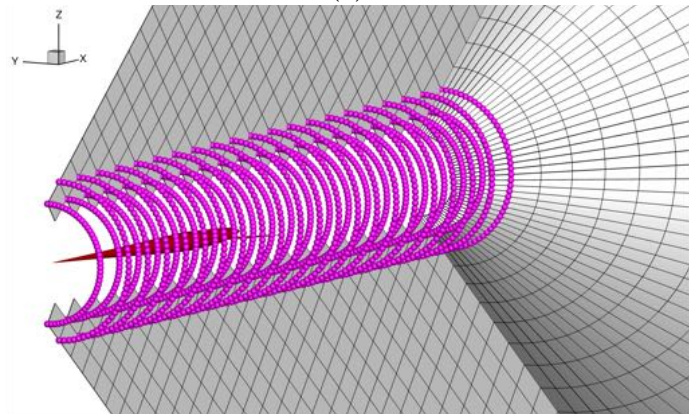

(h)

Figure 2. Step-by-step diagram of the autmatically generated space marching grid. (a) Initial rectangle based on length $L$ of the inner cylinder and Mach cone angle $\mu$ based on the free-stream Mach number $M_{\infty}$. (b) Discretization of the initial rectangle using a diagonal length $\Delta s=L /\left(N_{s t r m}-1\right)$ where $N_{s t r m}$ is the number of grid points in the streamwise direction. (c) Extension of the rectangle using the stretching ratio $S R$ and maximum aspect ratio $A R$. (d) Rotation of the symmetry plane grid for Mach cone angle alignment. (e) Translation of the symmetry plane grid. (f) Blanking of the symmetry plane grid points with $\mathrm{z}$ coordinates above $-\mathbf{r}_{0}$. (g) Revolution of the symmetry plane grid to a full three-dimensional grid. (h) Marking of the fringe points which identifies where the CFD solution is interpolated onto the space marching grid. 


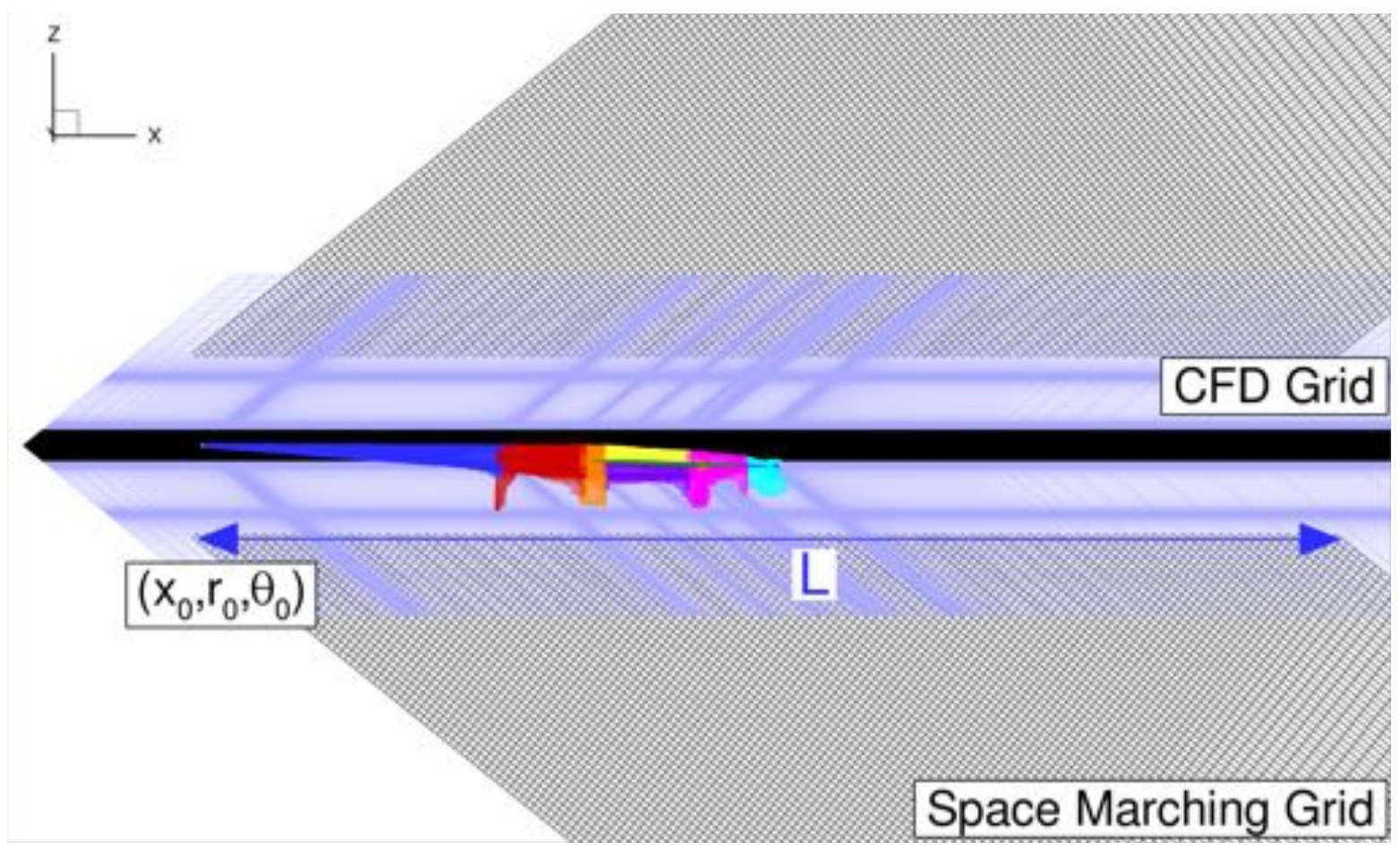

Figure 3. Symmetry plane view of the Mach-cone aligned cylindrical space marching grid and the structured overlapping CFD mesh illustrating that the inner cylinder of the space marching grid is contained within the CFD grid for proper solution interpolation.

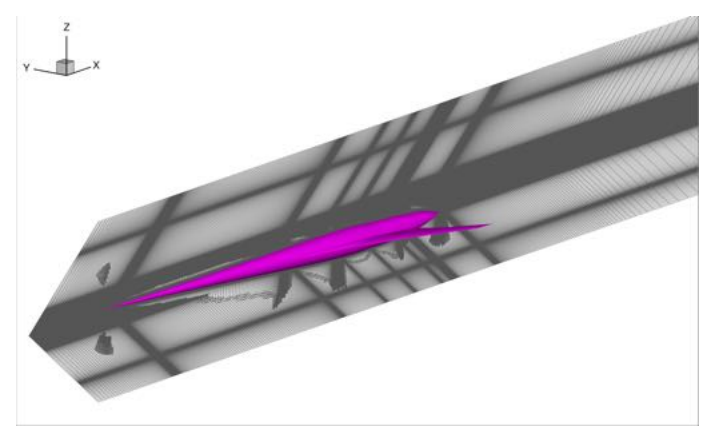

(a) CFD Mesh

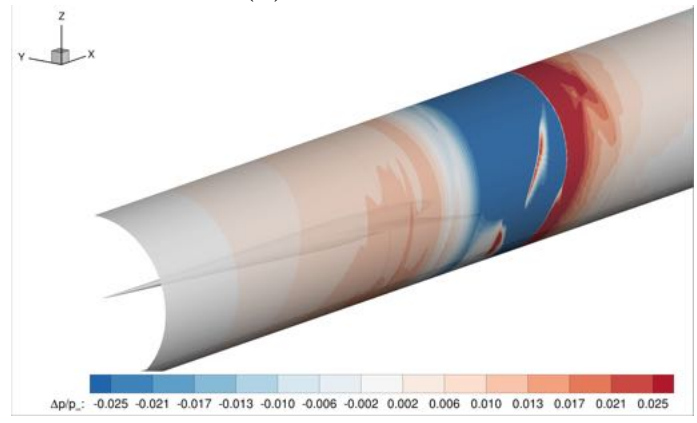

(c) Inner Cylinder

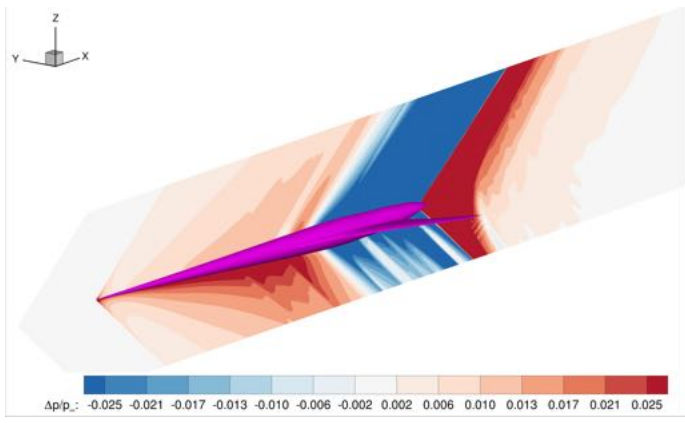

(b) CFD Solution

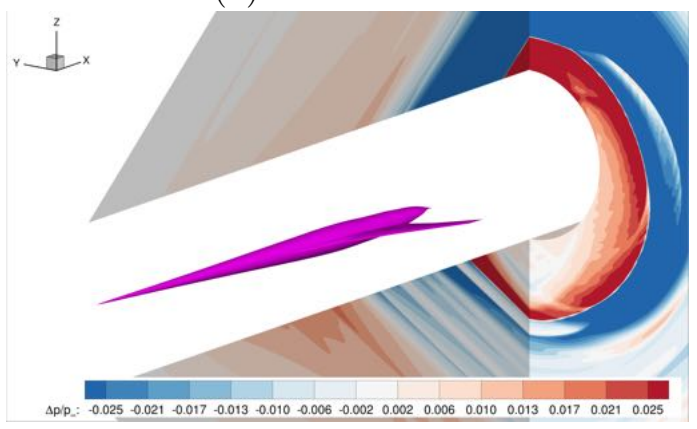

(d) Space March Solution

Figure 4. Diagram of the near-field to mid-field sonic boom progration procedure. (a) Generate the near-field CFD grid with a radial extent slighly larger than the semi-span of the aircraft. (b) Compute the steady-state RANS CFD solution on the near-field grid. (c) Interpolation the CFD solution onto the fringe points of the space marching grid. (d) Perform the single sweep space marching procedure to propagate the near-field solution to the mid-field. 

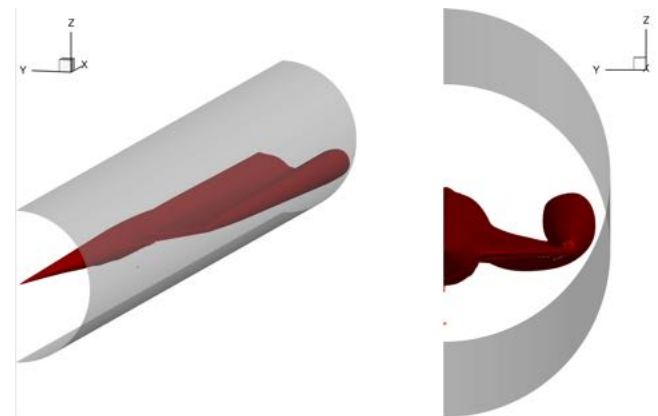

(a) Extraction Cylinder

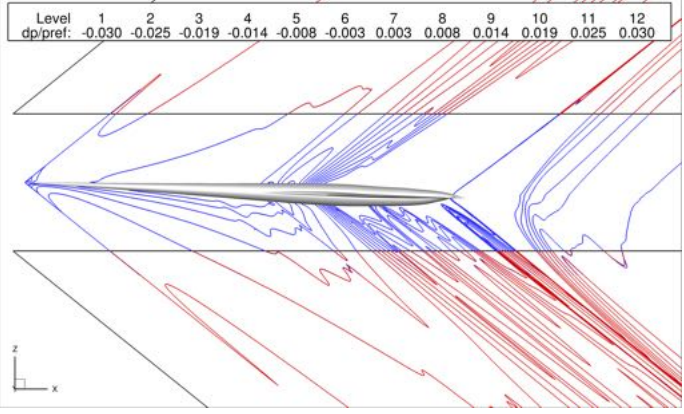

(c) Constant $Y=0 \mathrm{~m}$

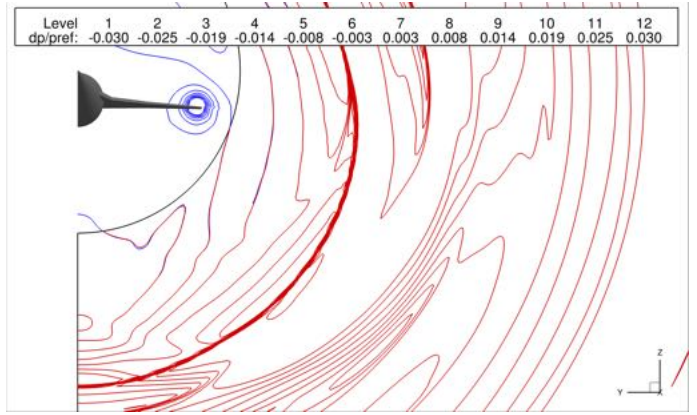

(b) Constant $X=50 \mathrm{~m}$

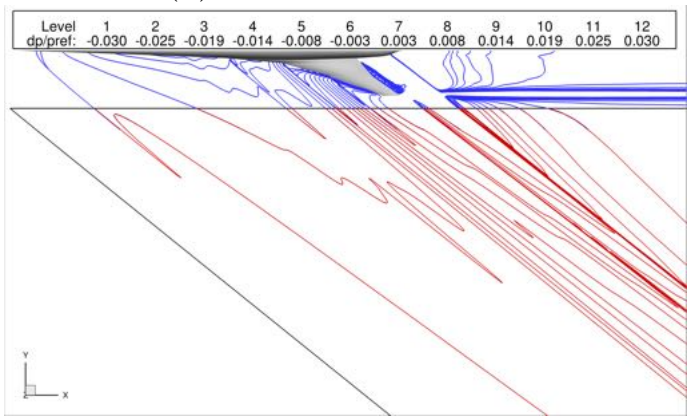

(d) Constant $Z=-1.3 \mathrm{~m}$

Figure 5. (a) Two views of the inner cylinder surface relative to an iso-surface of turbulent eddy viscosity ratio $\mu_{T} / \mu_{r e f}=10$. Contour lines of the CFD (blue) and space marching solution (red) at a constant $X=50 \mathrm{~m}$ (b), a constant $Y=0 \mathbf{m}(\mathbf{c})$, and a constant $Z=-1.3 \mathbf{m}(\mathbf{d})$.

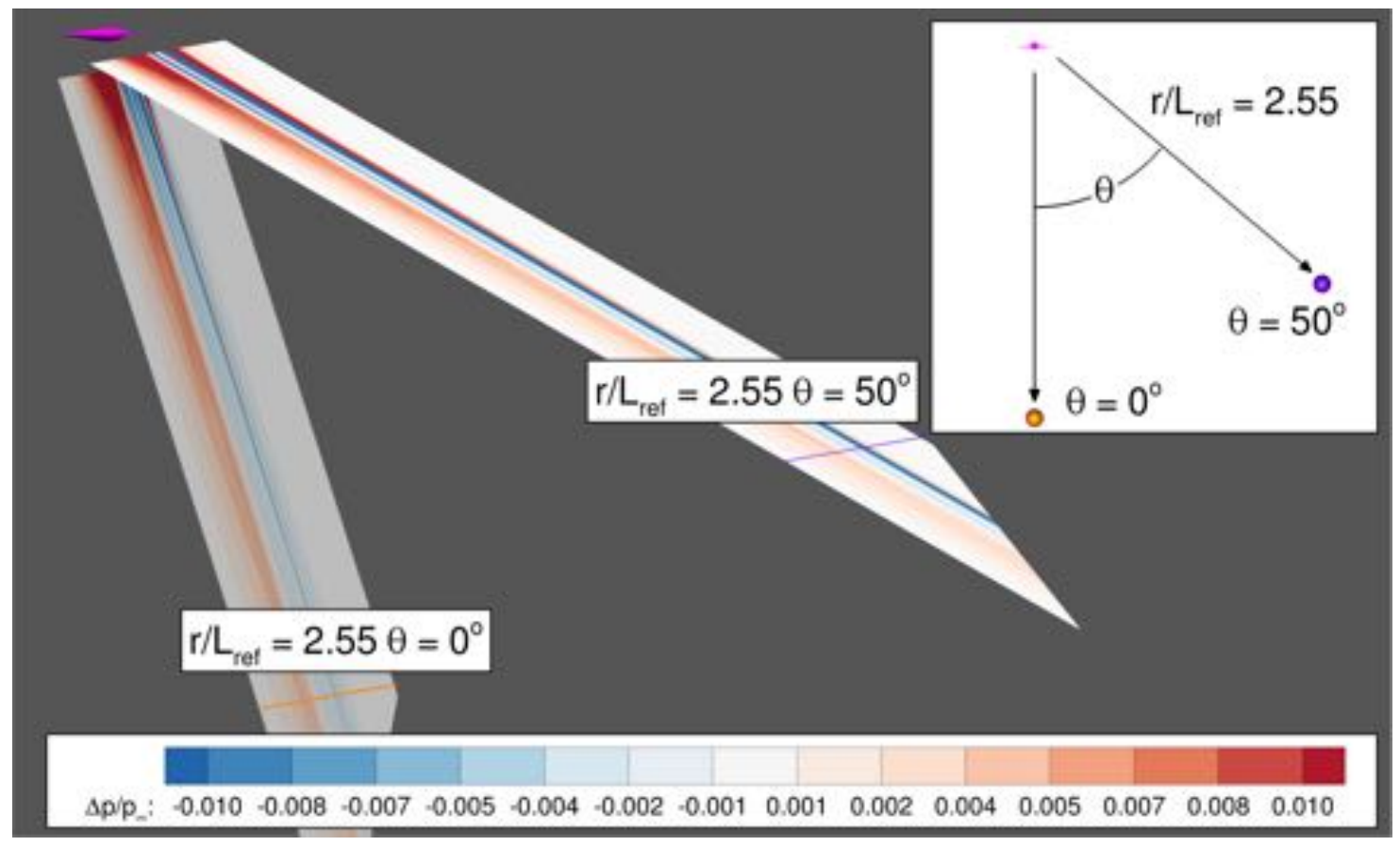

Figure 6. Diagram showing axial line extraction locations for the space marching sensitivity studies. These locations include $r / L_{\text {ref }}=2.55, \theta=0^{\circ}$ and $r / L_{\text {ref }}=2.55, \theta=\mathbf{5 0}^{\circ}$. 


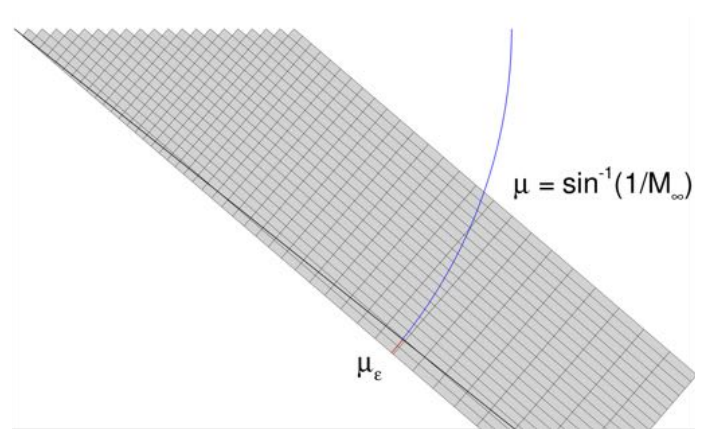

(a)

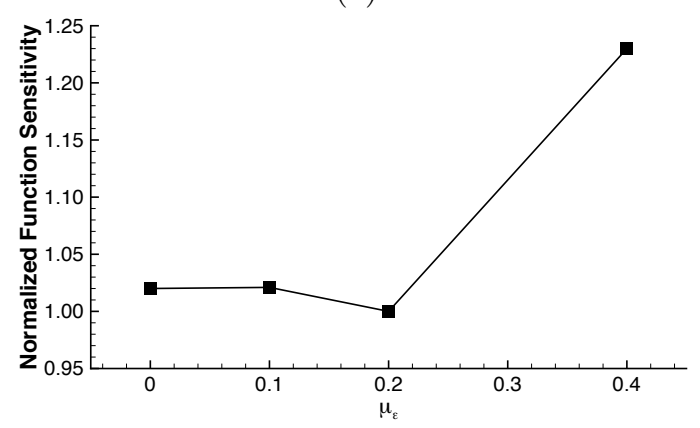

(c)

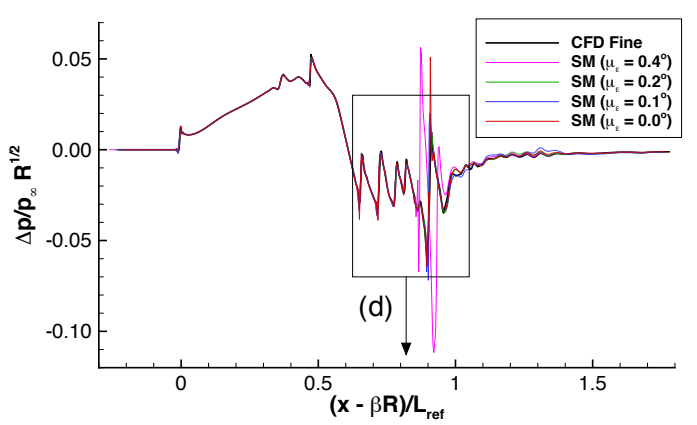

(b)

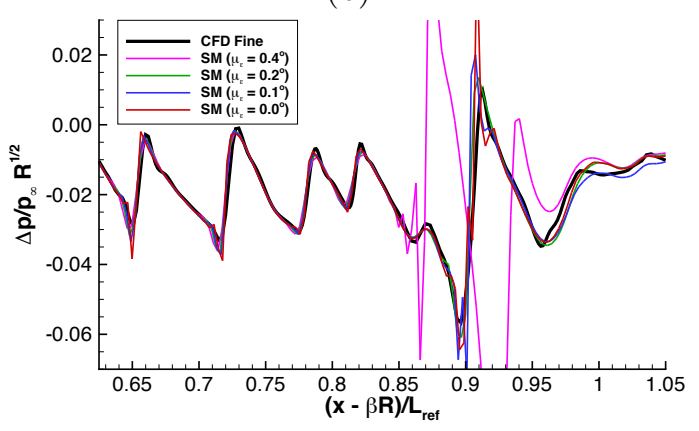

(d)

Figure 7. (a) Diagram illustrating the effect of the Mach-cone perturbation, $\mu_{\epsilon}$ on the space marching grid. (b) Comparison of the pressure signature at $r / L_{\text {ref }}=2.55, \theta=0^{\circ}$ between the CFD and space marching solution for each of the Mach-cone perturbation angles. (c) Functional sensitivity to $\mu_{\epsilon}$. (d) Close-up view of pressure comparison.

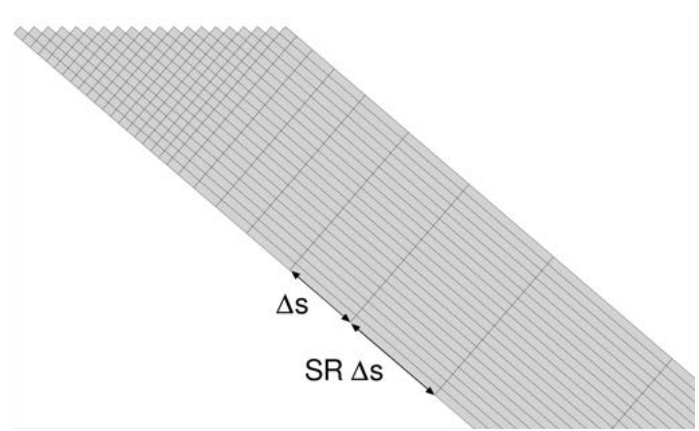

(a)

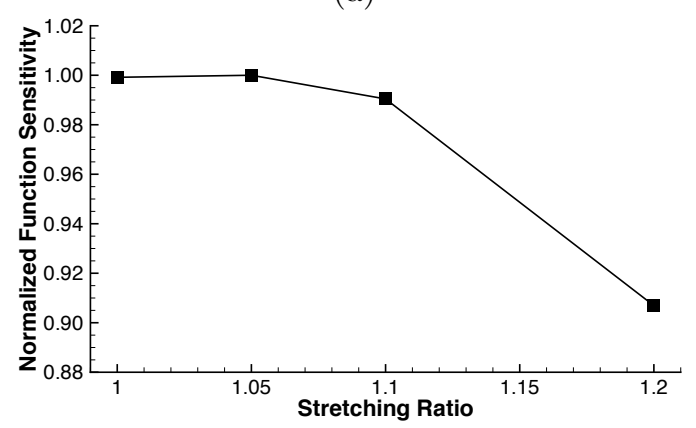

(c)

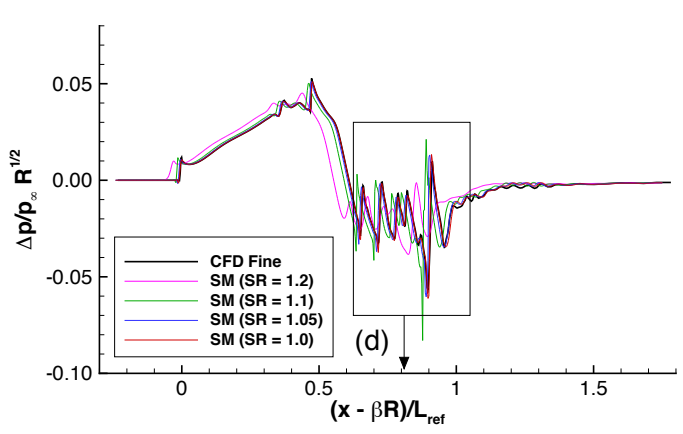

(b)

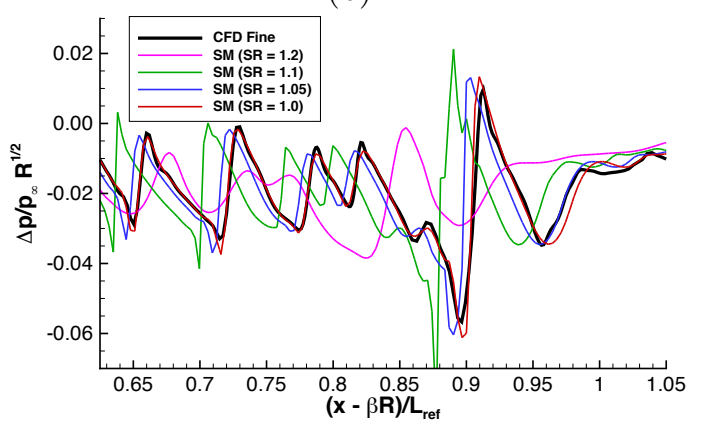

(d)

Figure 8. (a) Diagram illustrating the effect of stretching ratio on the space marching grid. (b) Comparison of the pressure signature at $\mathrm{r} / \mathrm{L}_{\text {ref }}=\mathbf{2 . 5 5}, \theta=0^{\circ}$ between the CFD and space marching solution for four different stretching ratios. (c) Functional sensitivity to stretching ratio. (d) Close-up view of pressure comparison. 


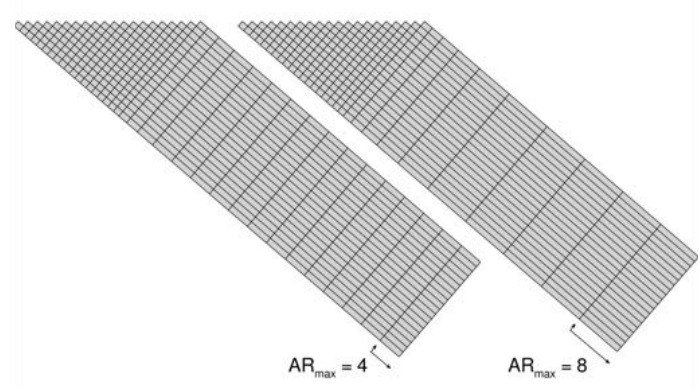

(a)

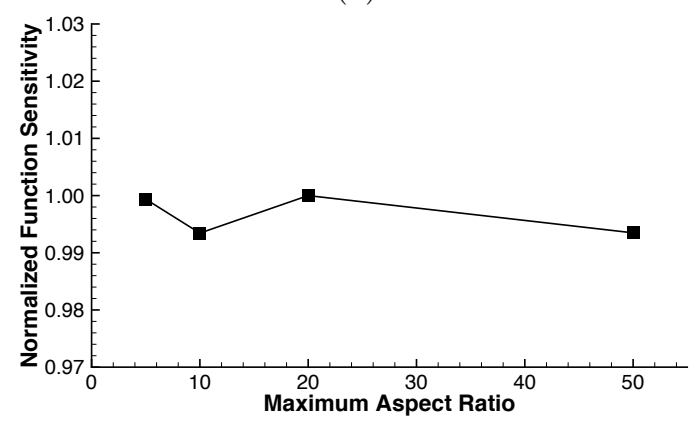

(c)

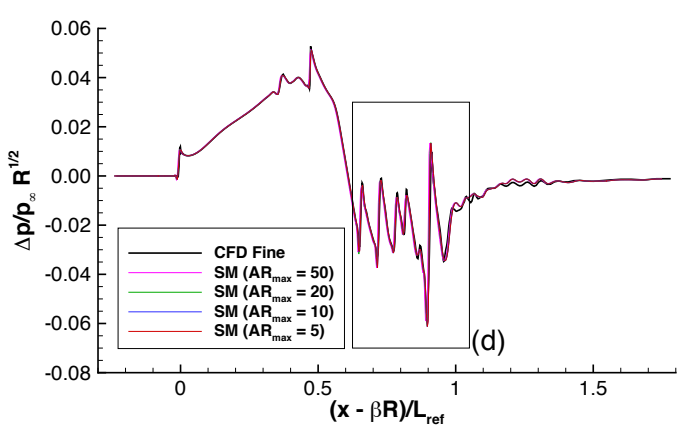

(b)

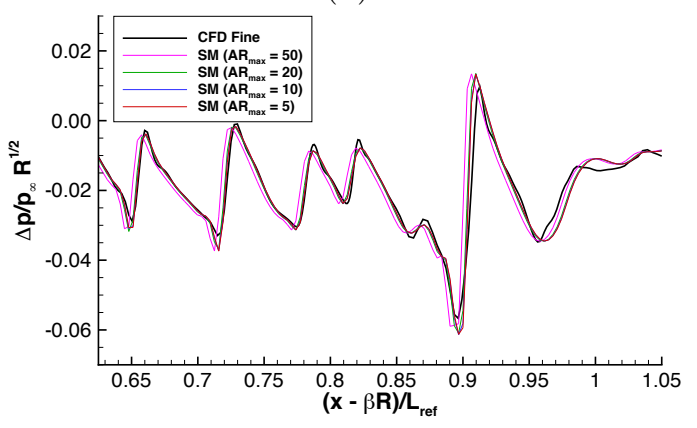

(d)

Figure 9. (a) Diagram illustrating the effect of maximum aspect ratio on the space marching grid. (b) Comparison of the pressure signature at $r / L_{\text {ref }}=2.55, \theta=0^{\circ}$ between the CFD and space marching solution for four different maximum aspect ratios. (c) Functional sensitivity to maximum aspect ratio. (d) Close-up view of pressure comparison.

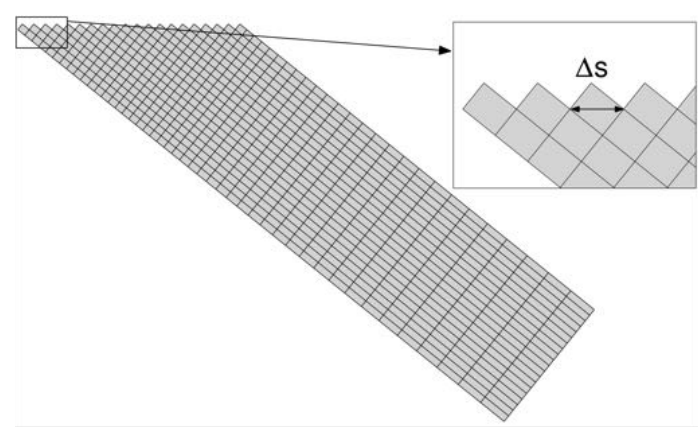

(a)

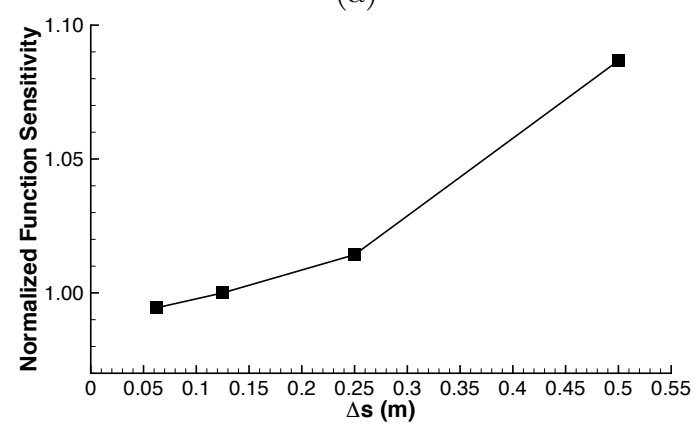

(c)

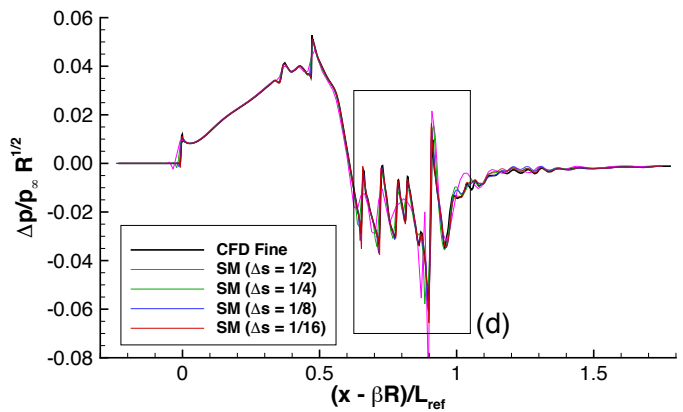

(b)

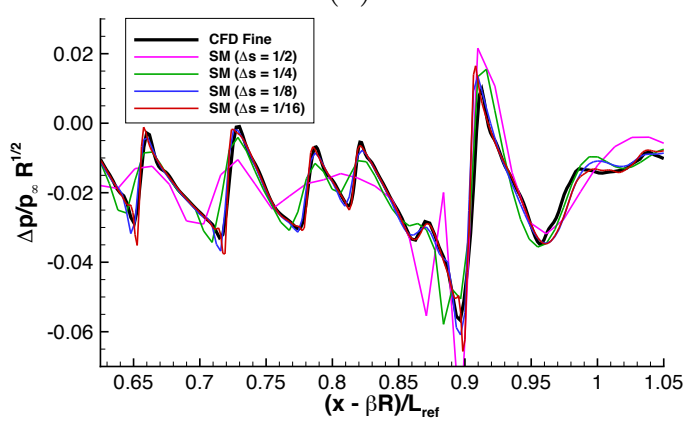

(d)

Figure 10. (a) Diagram illustrating the streamwise spacing parameter on the space marching grid. (b) Comparison of the pressure signature at $r / L_{\text {ref }}=2.55, \theta=0^{\circ}$ between the CFD and space marching solution for four different streamwise spacings. (c) Functional sensitivity to streamwise spacing. (d) Close-up view of pressure comparison. 


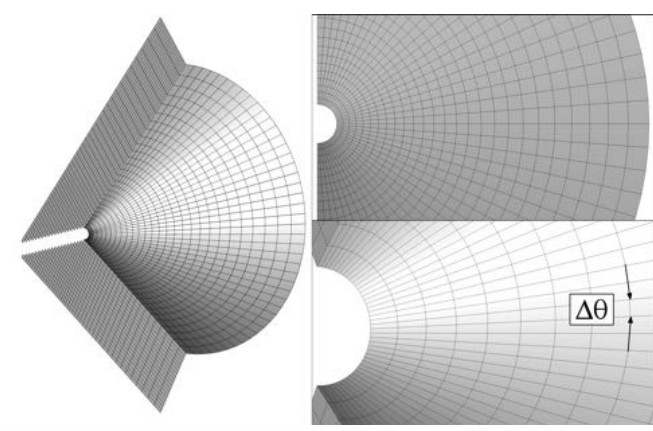

(a)

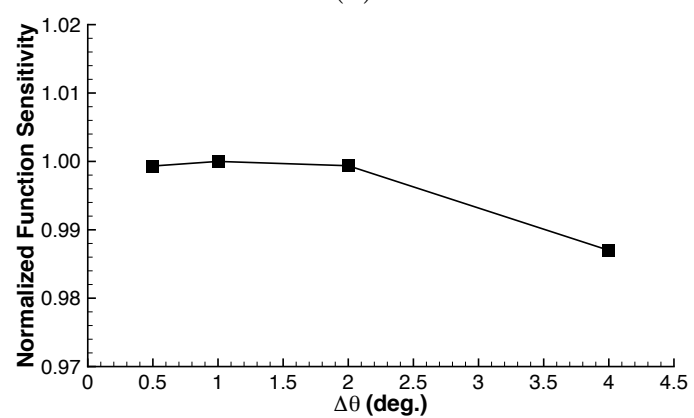

(c)

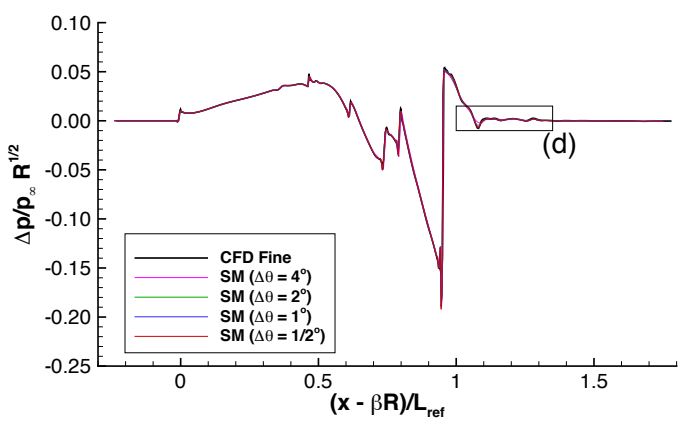

(b)

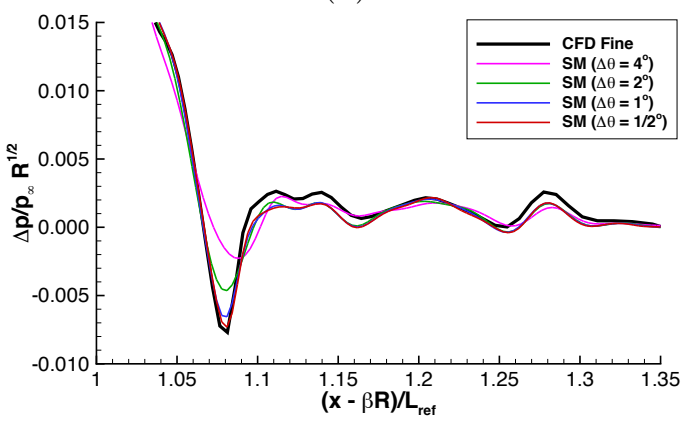

(d)

Figure 11. (a) Diagram illustrating the circumferential spacing parameter on the space marching grid. (b) Comparison of the pressure signature at $\mathrm{r} / \mathrm{L}_{\mathrm{ref}}=\mathbf{2 . 5 5}, \theta=\mathbf{5 0}^{\circ}$ between the CFD and space marching solution for four different circumferential spacings. (c) Functional sensitivity to circumferential spacing parameter. (d) Close-up view of pressure comparison.

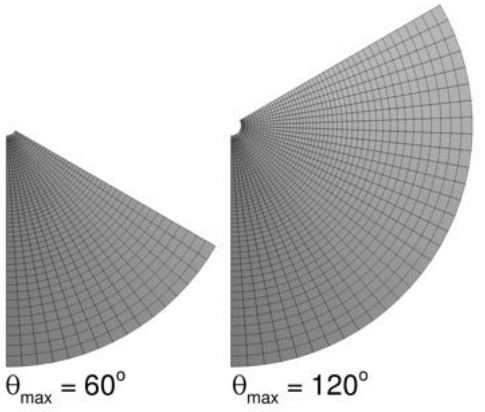

(a)

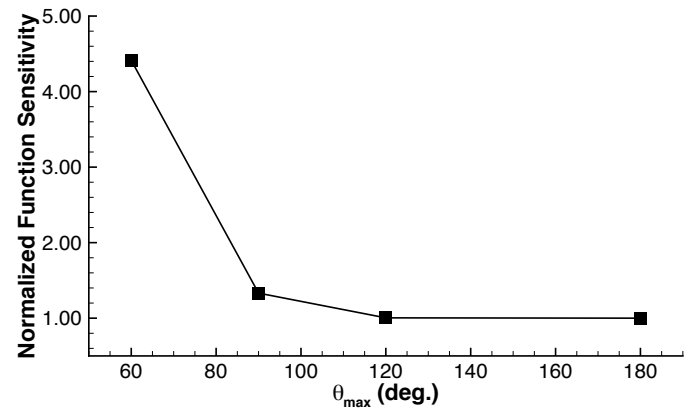

(c)

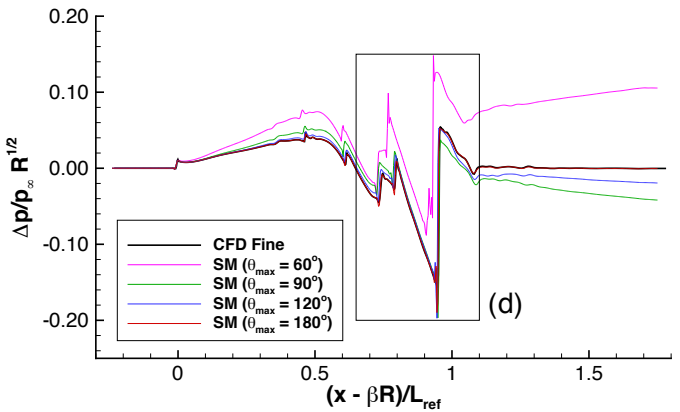

(b)

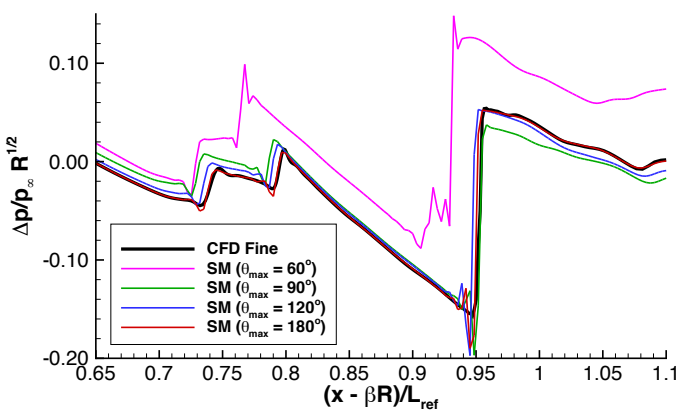

(d)

Figure 12. (a) Diagram illustrating the effect of the $\theta$ max parameter on the space marching grid. (b) Comparison of the pressure signature at $\mathrm{r} / \mathrm{L}_{\text {ref }}=\mathbf{2 . 5 5}, \theta=50^{\circ}$ between the CFD and space marching solution for four different $\theta$ max domain extents. (c) Functional sensitivity to $\theta$ max parameter. (d) Close-up view of pressure comparison. 


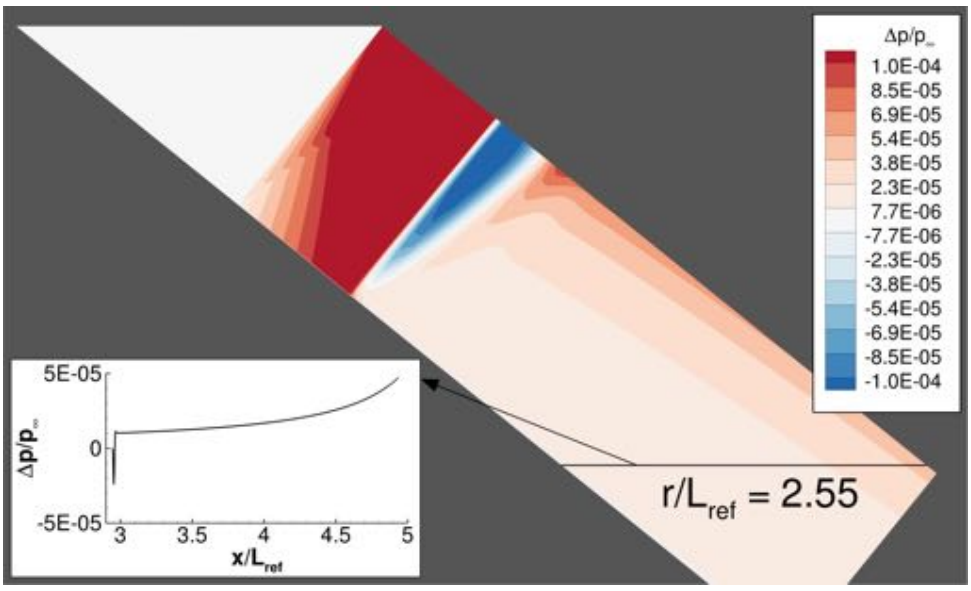

(a) Standard Metics

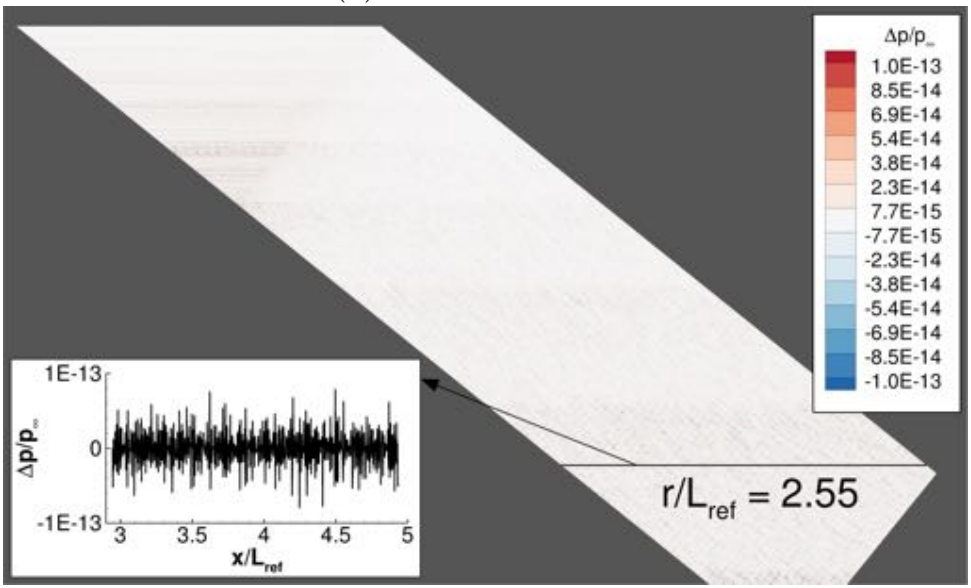

(b) Conservative Metric Method

Figure 13. Results of the free-stream preservation test applied to the space marching scheme using (a) standard metric term evaluation and (b) the conservative metric method.

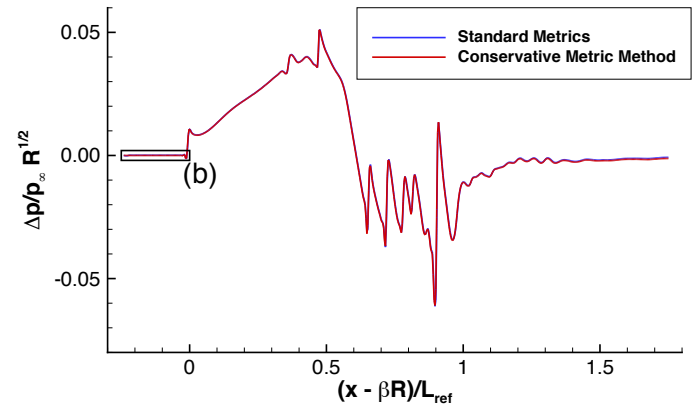

(a)

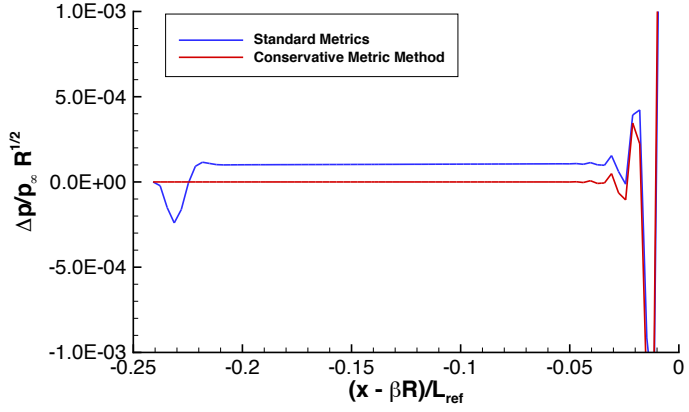

(b)

Figure 14. (a) Comparison of the pressure signature at $\mathbf{r} / \mathbf{L}_{\text {ref }}=2.55, \theta=0^{\circ}$ predicted by the space marching scheme using standard metric term evaluation and the conservative metric method over the entire signature. (b) Close-up of the signature comparison at the front of the signature. 


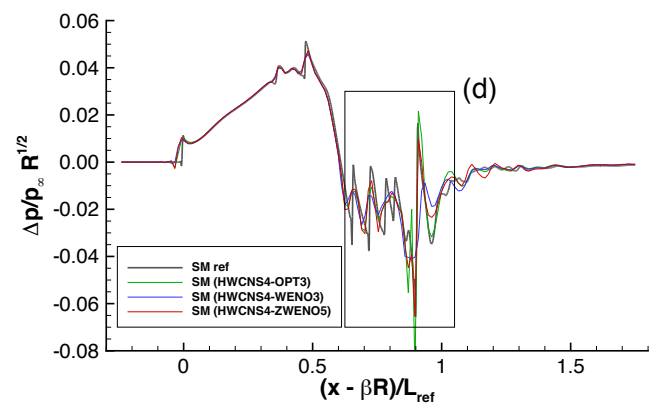

(a) Coarse grid: $\Delta s=1 / 2 \mathrm{~m}$

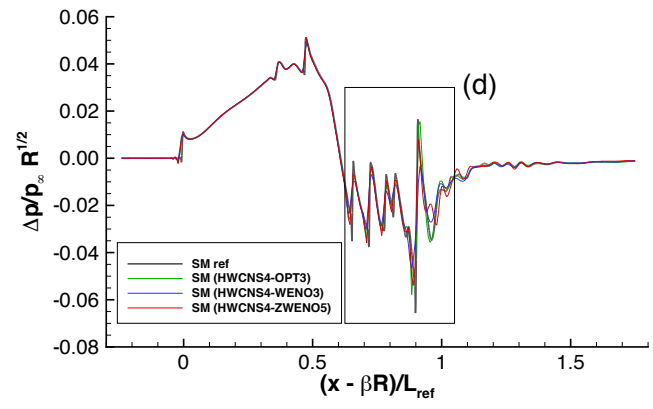

(c) Medium grid: $\Delta s=1 / 4 \mathrm{~m}$

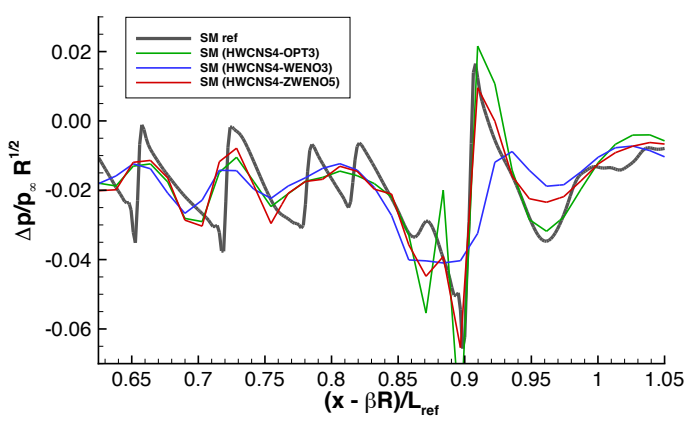

(b) Zoom-in of rectangular box

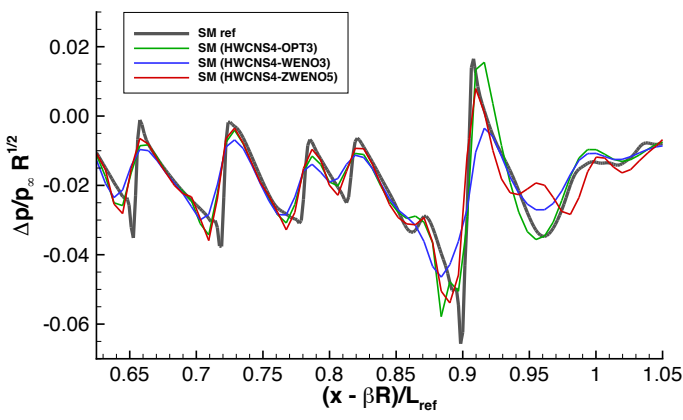

(d) Zoom-in of rectangular box

Figure 15. Comparison of the pressure signature at $\mathbf{r} / \mathrm{L}_{\mathrm{ref}}=\mathbf{2 . 5 5}, \theta=0^{\circ}$ between the CFD, the reference space marching solution on the finest grid $\Delta \mathrm{s}=1 / 16 \mathrm{~m}$, and the space marching solution using four different convective flux discretization on the coarse and medium mesh resolutions.

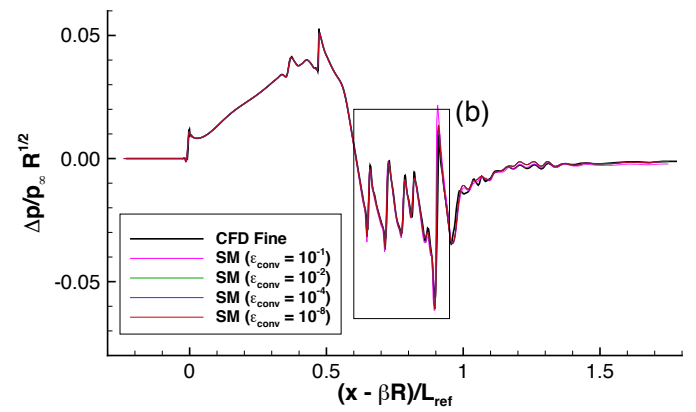

(a)

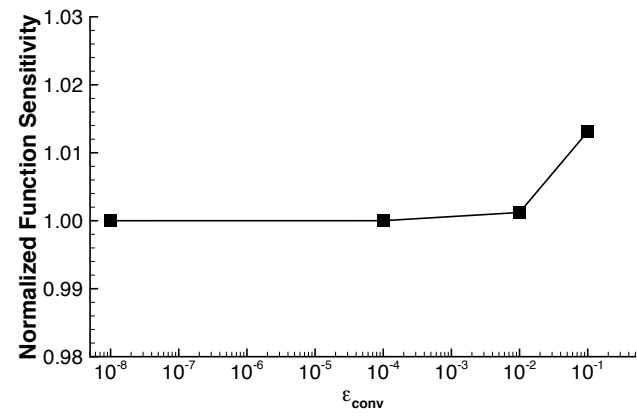

(c)

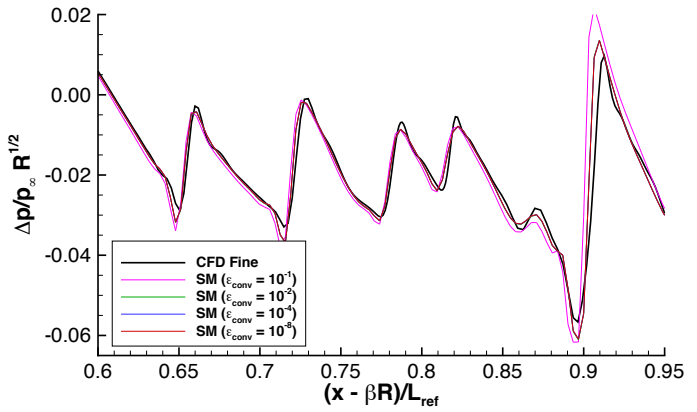

(b)

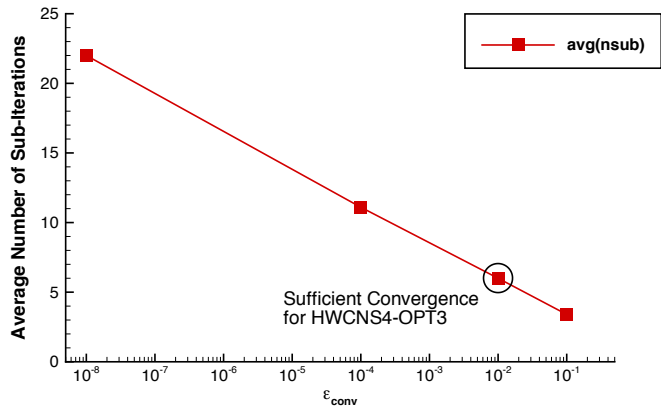

(d)

Figure 16. (a) Comparison of the pressure signature at $r / L_{\text {ref }}=2.55, \theta=0^{\circ}$ between the CFD and the space marching solution (HWCNS4-OPT3) using four different residual convergence tolerances, $\epsilon$ conv. (b) Close-up view of the pressure signature. (c) Functional sensitivity to residual convergence tolerance. (d) Average number of sub-iterations required to meet the convergence tolerance plotted as a function of the convergence tolerance. 


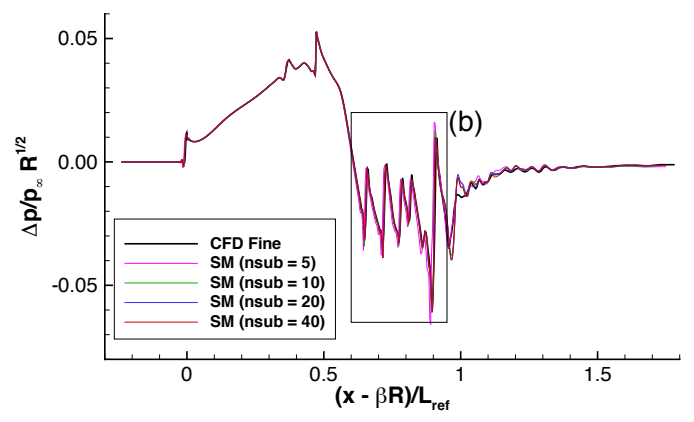

(a)

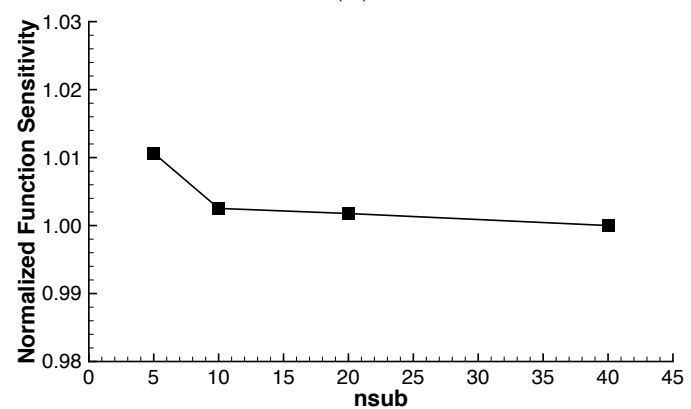

(c)

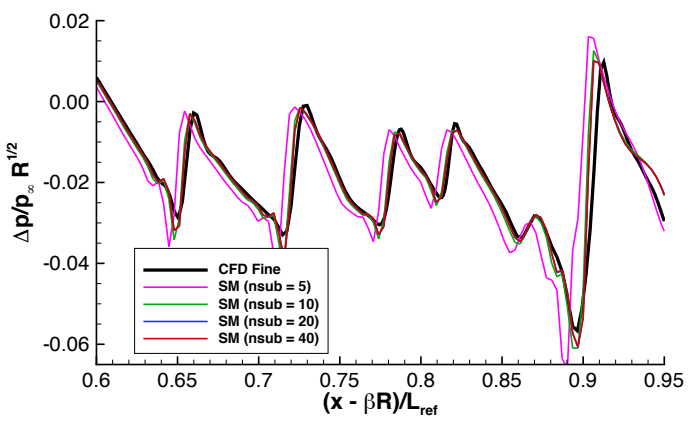

(b)

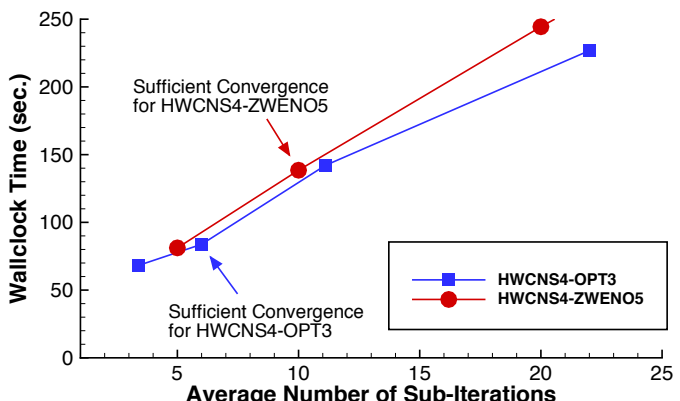

(d)

Figure 17. (a) Comparison of the pressure signature at $\mathbf{r} / \mathbf{L}_{\text {ref }}=\mathbf{2 . 5 5}, \theta=0^{\circ}$ between the CFD and the space marching solution (HWCNS5-ZWENO5) using four different number of sub-iterations at each space marching station, $\epsilon_{\text {conv }}$. (b)

Close-up view of the pressure signature. (c) Functional sensitivity to residual convergence tolerance. (d) Comparison of wall-clock time as a function of average number of sub-iterations for HWCNS4-OPT3 and HWCNS4-ZWENO5.

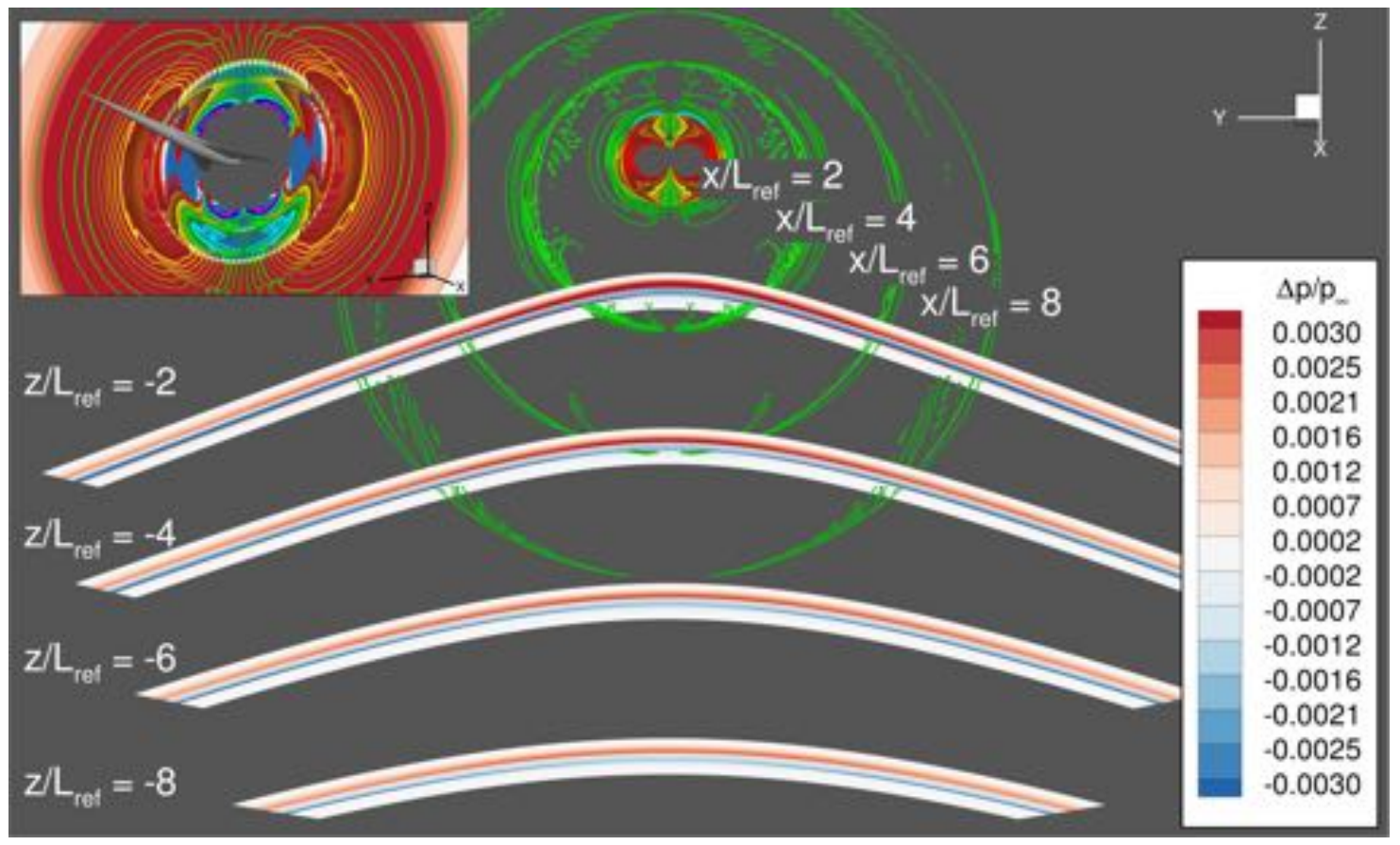

Figure 18. Contour lines of azimuthal velocity a constant streamwise slices of $x / L$ ref $=2,4,6,8$ along with contours of pressure at several elevations below the aircraft, $\mathrm{z} / \mathrm{L}_{\mathrm{ref}}=-2,-4,-6,-8$. Inset: Close-up view of the azimuthal velocity contour lines plotted on top of the pressure contours at a constant streamwise plane behind the aircraft. 


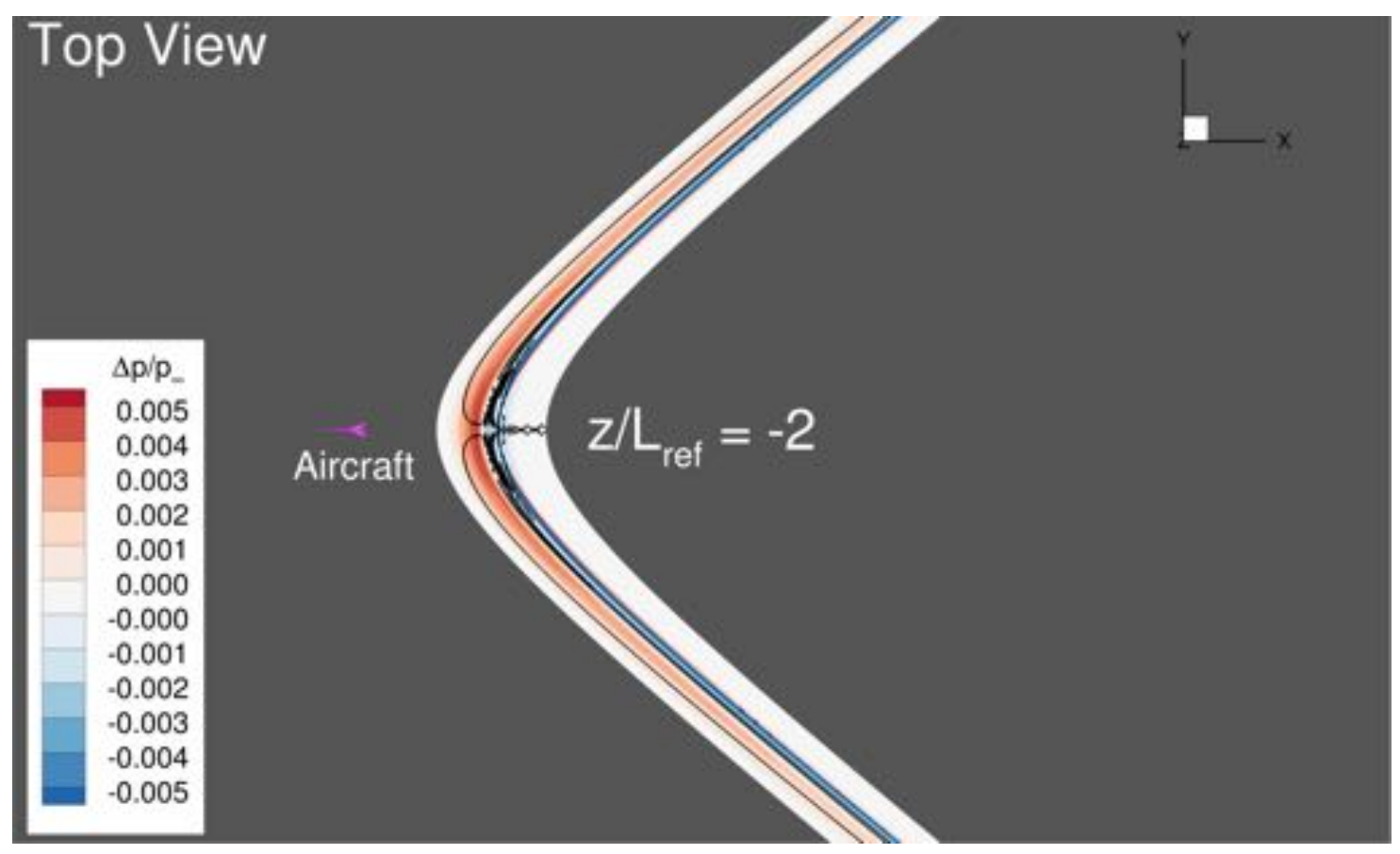

Figure 19. Contour plot of pressure with lines of azimuthal velocity magnitude at $\mathrm{z} / \mathrm{L}_{\mathrm{ref}}=-2$ showing the contentration of azimuthal velocity focused on the shock/expansion generated by the wing.

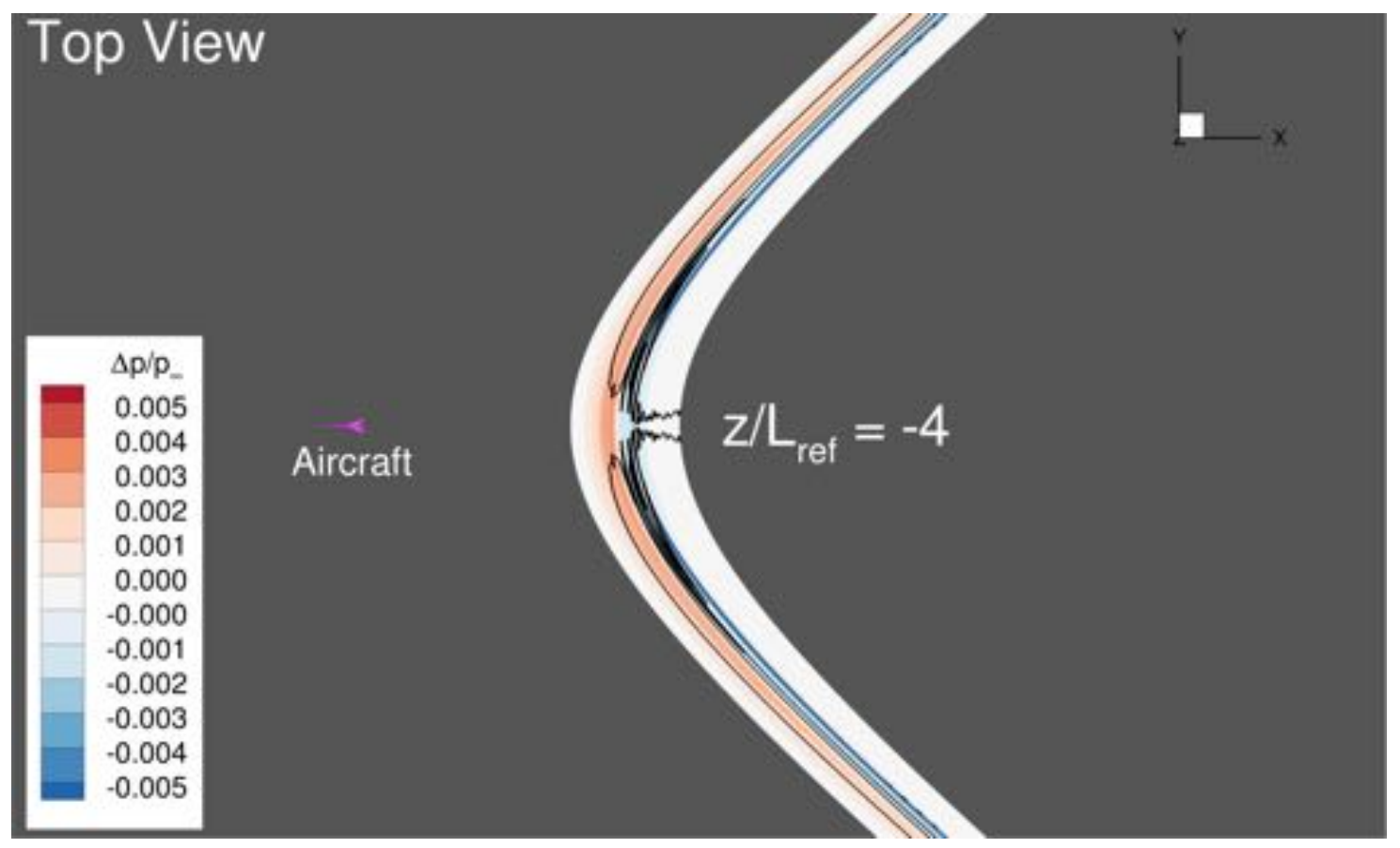

Figure 20. Contour plot of pressure with lines of azimuthal velocity magnitude at $\mathrm{z} / \mathrm{L}_{\mathrm{ref}}=-4$ showing the contentration of azimuthal velocity focused on the shock/expansion generated by the wing. 


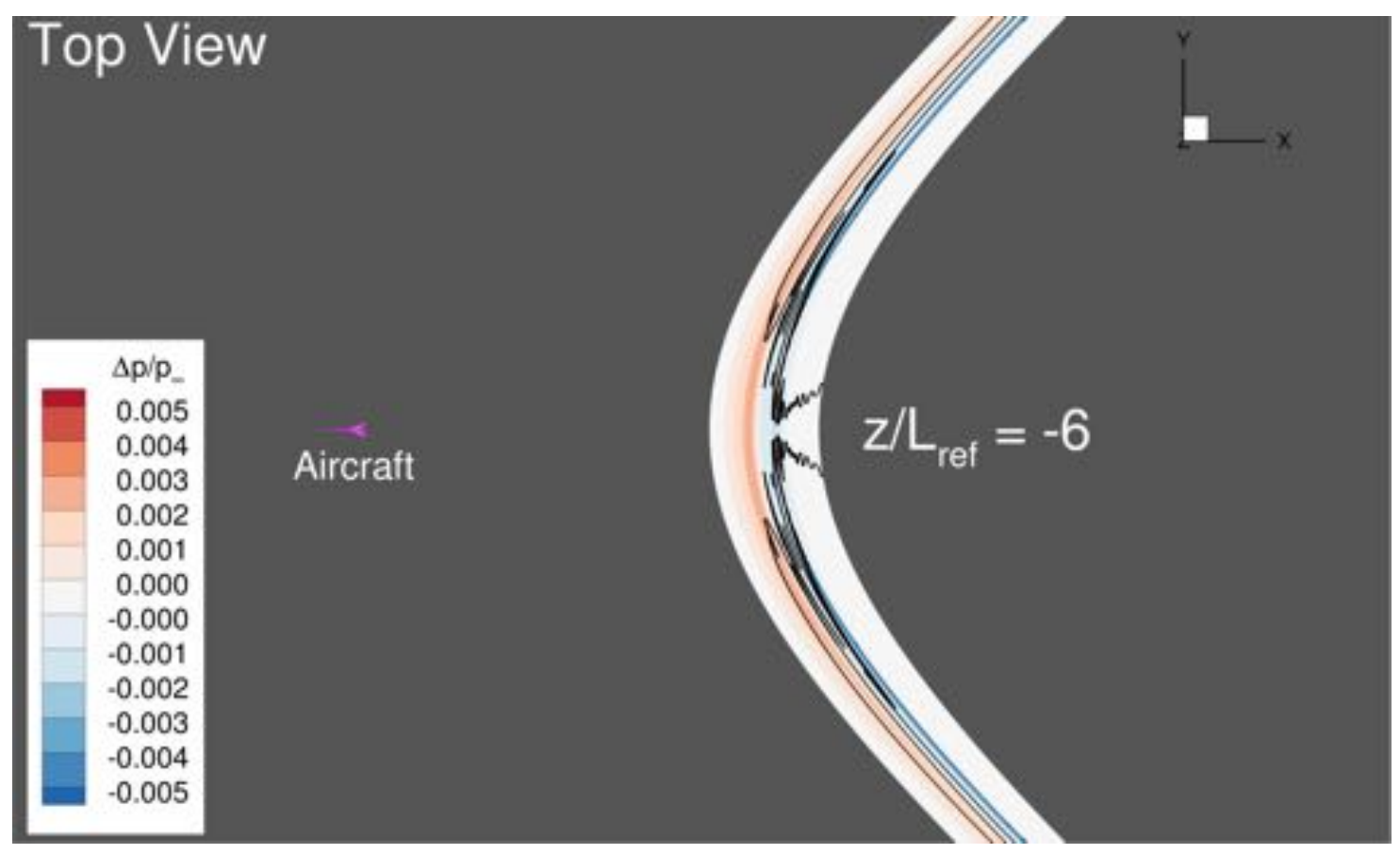

Figure 21. Contour plot of pressure with lines of azimuthal velocity magnitude at $\mathrm{z} / \mathrm{L}_{\mathrm{ref}}=-6$ showing the contentration of azimuthal velocity focused on the shock/expansion generated by the wing.

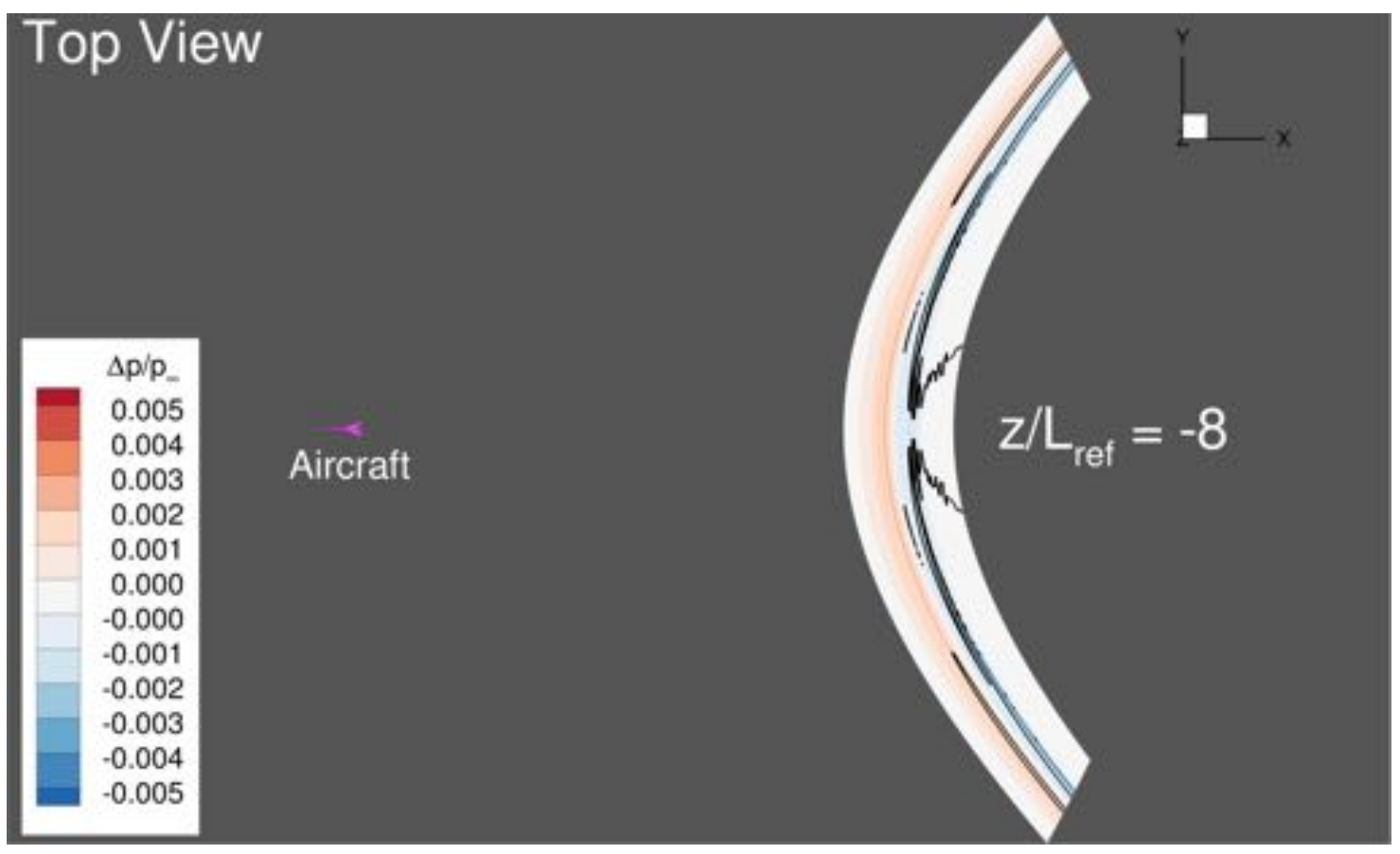

Figure 22. Contour plot of pressure with lines of azimuthal velocity magnitude at $\mathrm{z} / \mathrm{L}_{\mathrm{ref}}=-8$ showing the contentration of azimuthal velocity focused on the shock/expansion generated by the wing. 


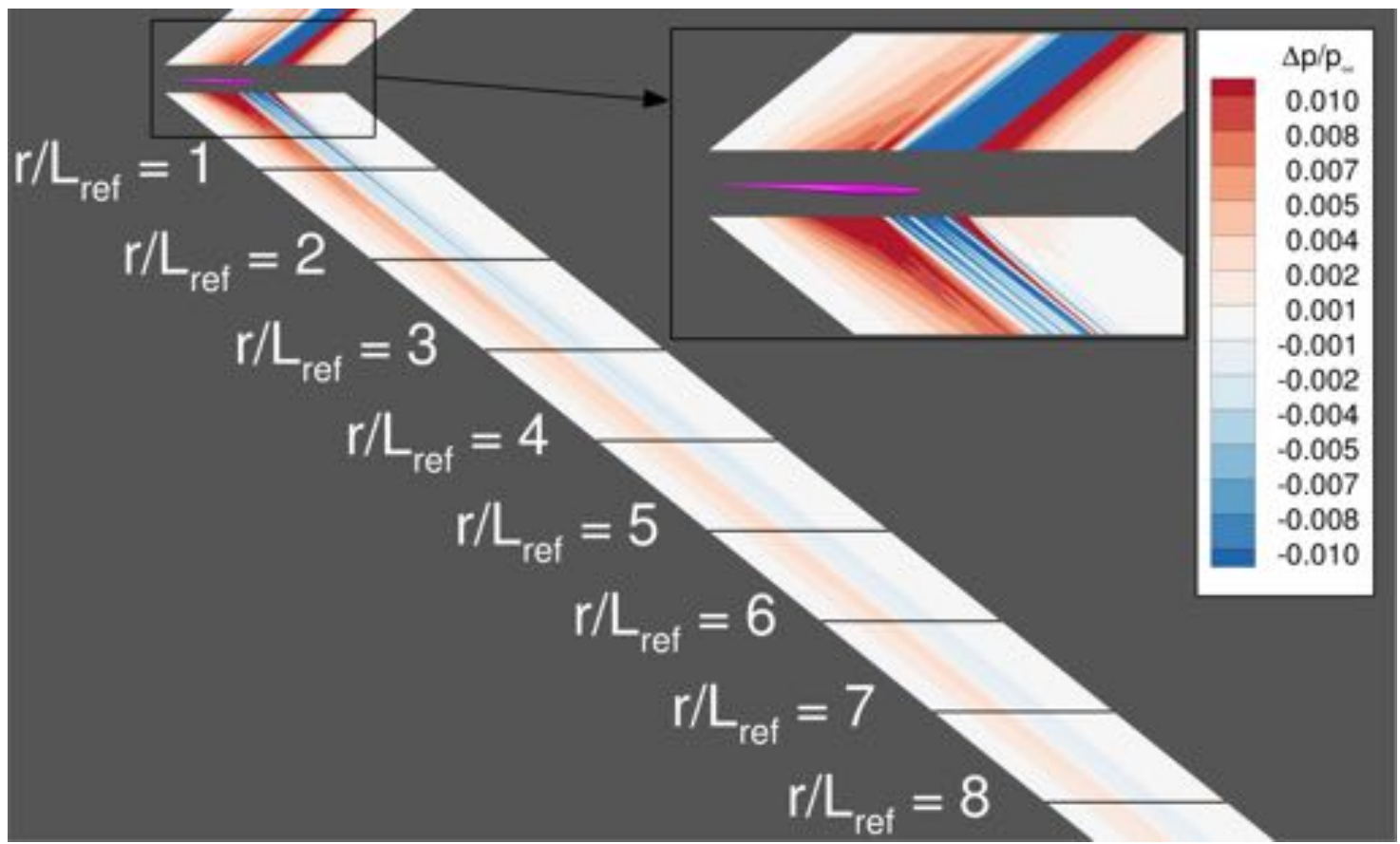

Figure 23. Diagram of on-track radial extraction lines from $r / L_{\text {ref }}=1$ to $r / L_{\text {ref }}=8$ shown on top of pressure contours on the symmetry below the aircraft.

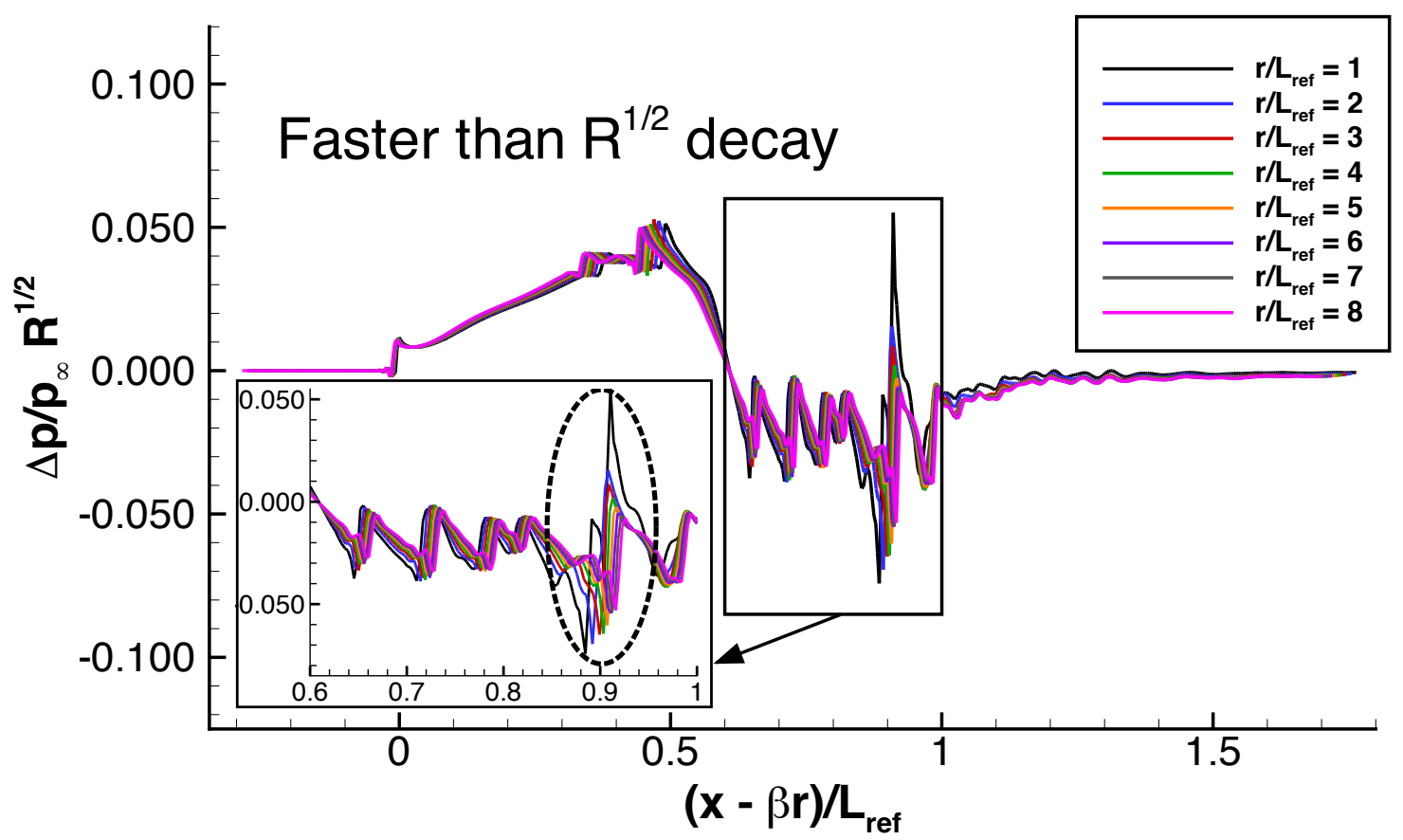

Figure 24. Normalized pressure signatures plotted for each radial extraction distance as a function of $(x-\beta r) / L_{\text {ref }}$. Inset: Close-up view of the pressure signature comparison showing the faster than $R^{1 / 2}$ decay of the shock/expansion generated by the wing. 


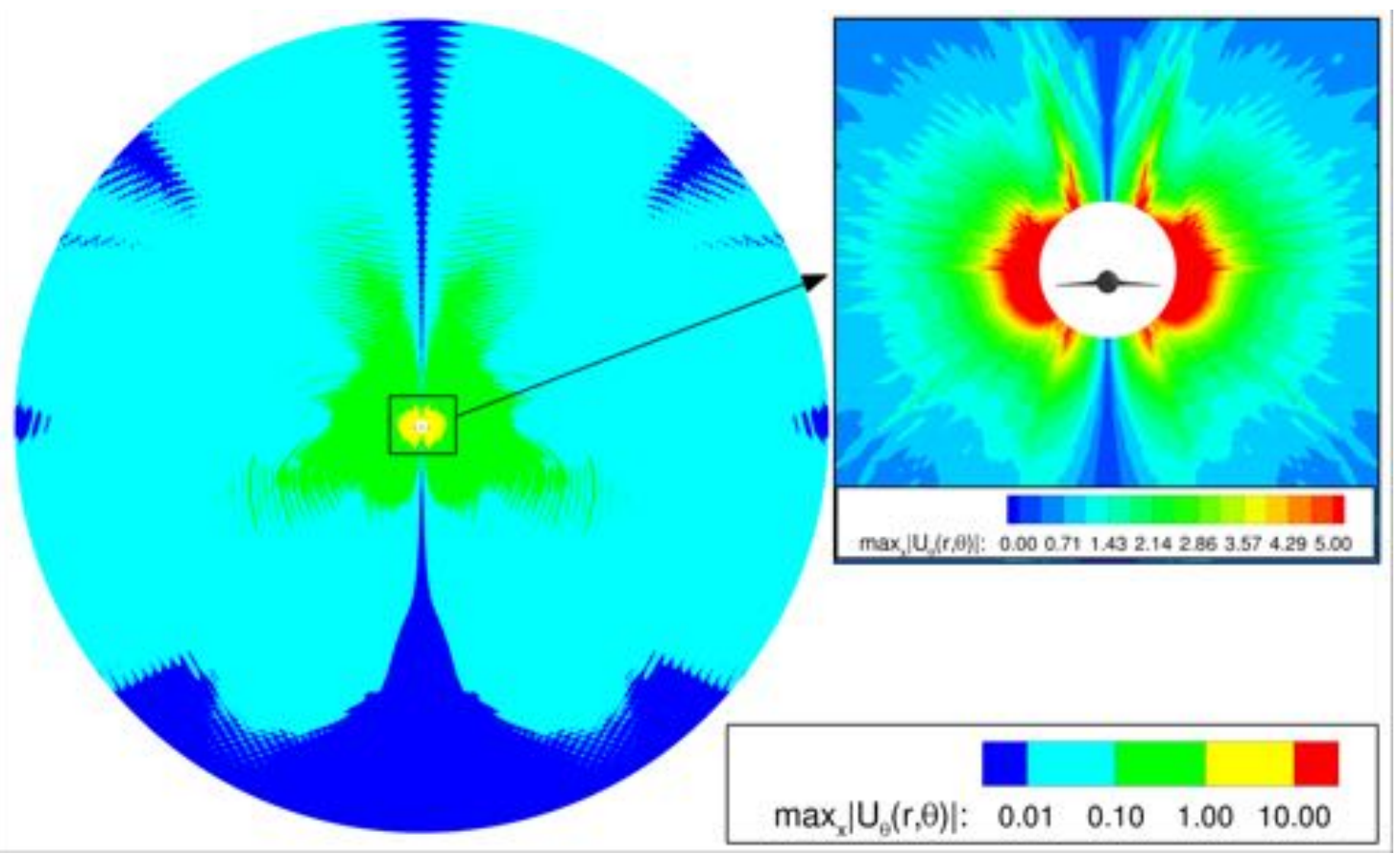

Figure 25. Contour plot of the maximum azimuthal velocity magnitude in the streamwise direction for each $\mathbf{r}, \theta$ showing the large radial extent of non-zero azimuthal velocity past 10 body lengths. Inset: Close-up view of the maximum azimuthal velocity magnitude near the aircraft.

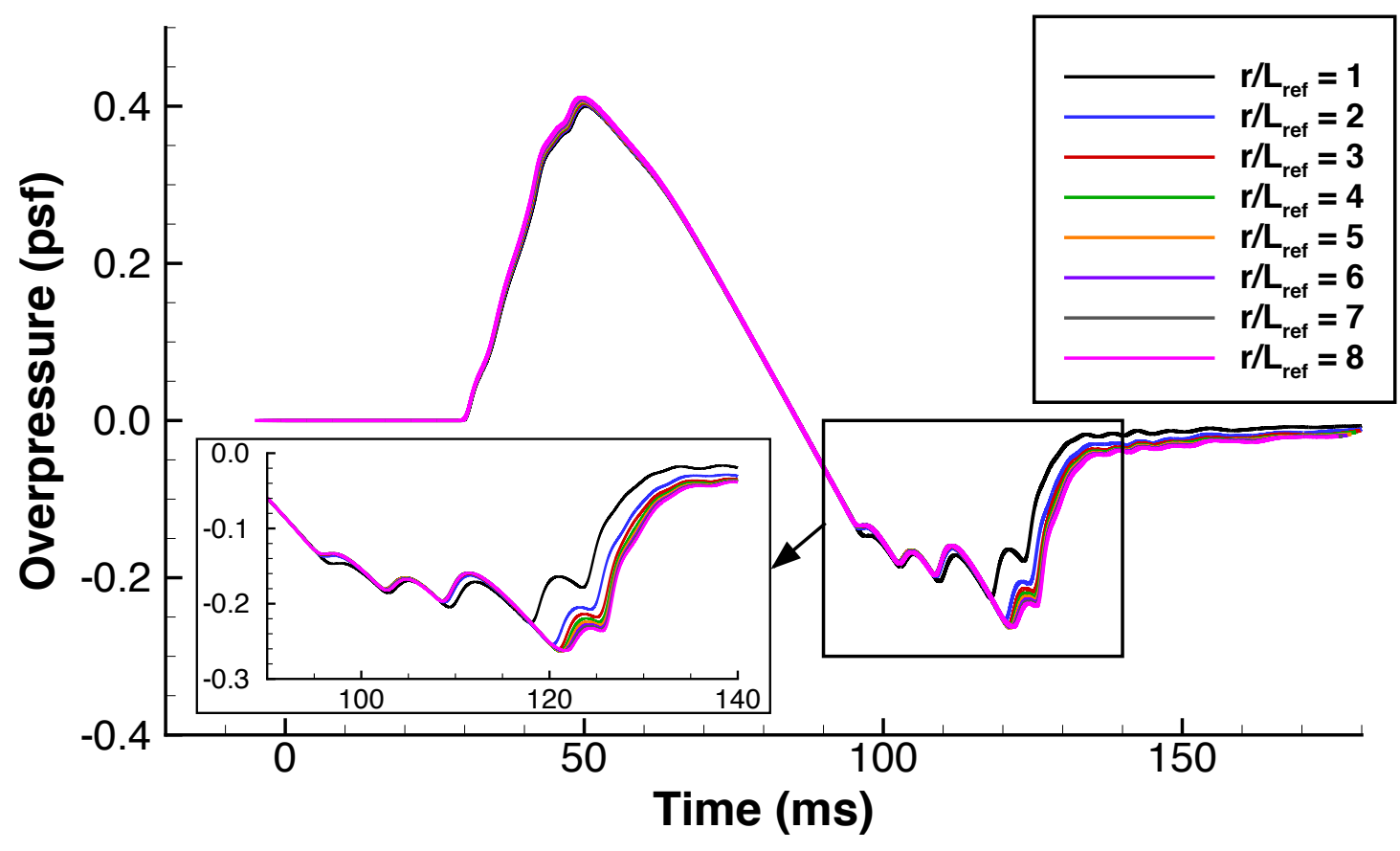

Figure 26. Overpressure ground signatures propagated from different radial extraction locations below the aircraft. Note that the ground signatures converge as the radial extraction location is increased. 


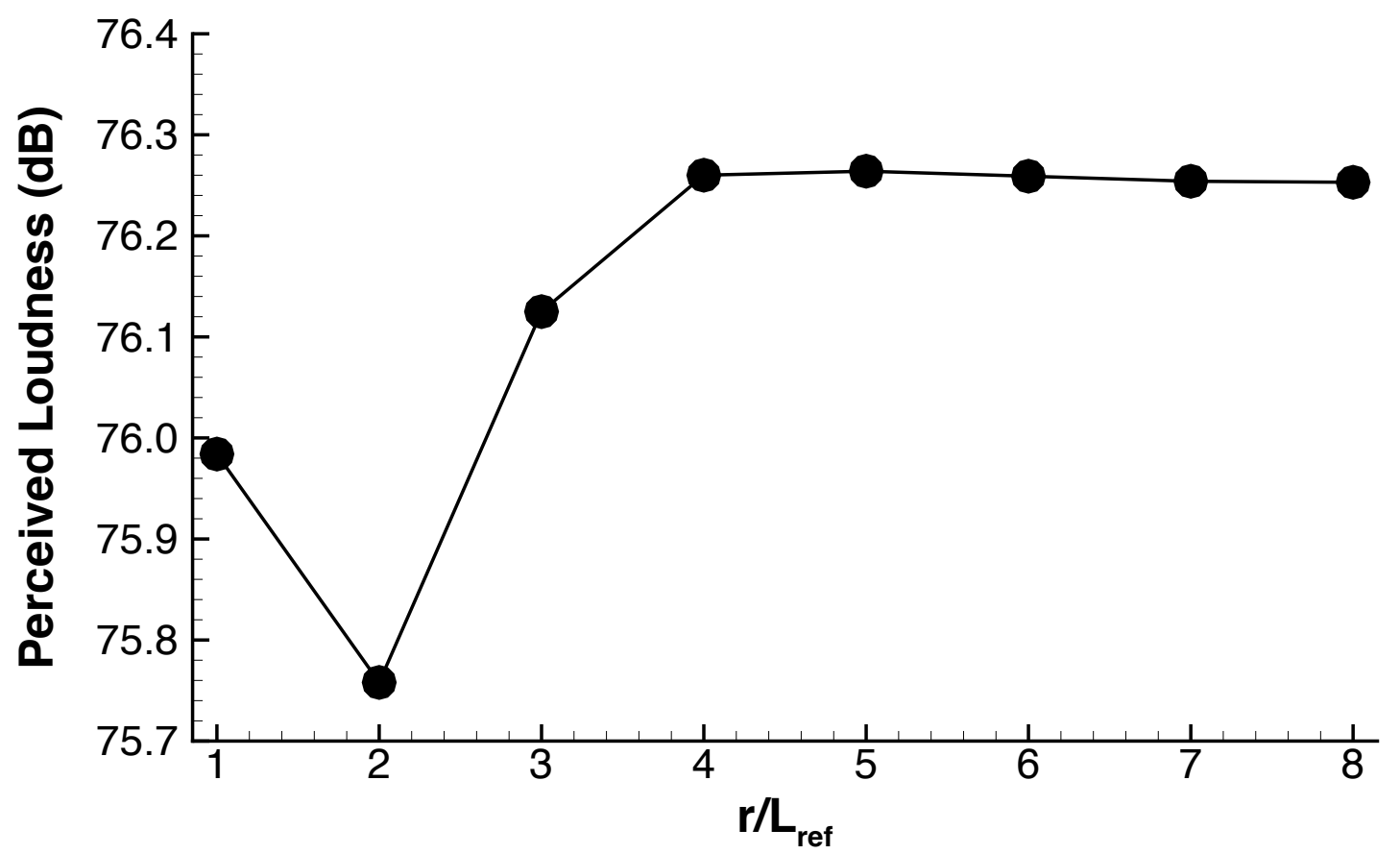

Figure 27. Percieved loudness metric plotted as a function of radial extraction location below the aircraft. A radial extraction distance of at least 4 body lengths is necessary for the loundness metric to converge.
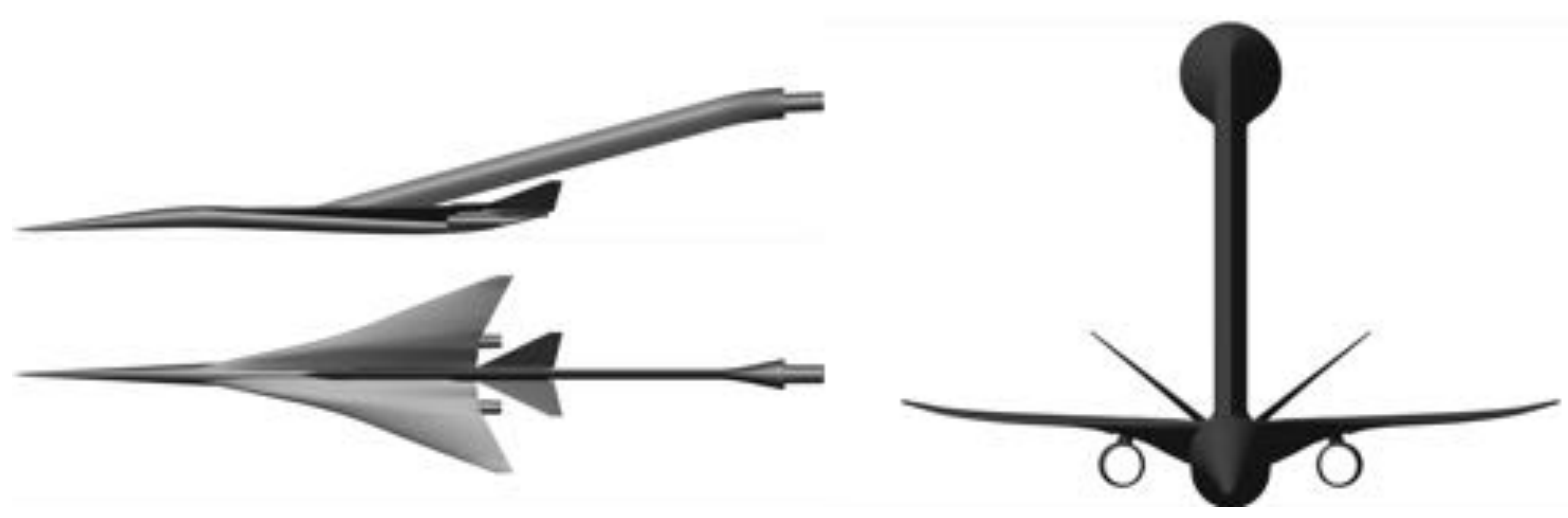

Figure 28. Three-view of the Lockheed Martin 1021 wind tunnel test article. 


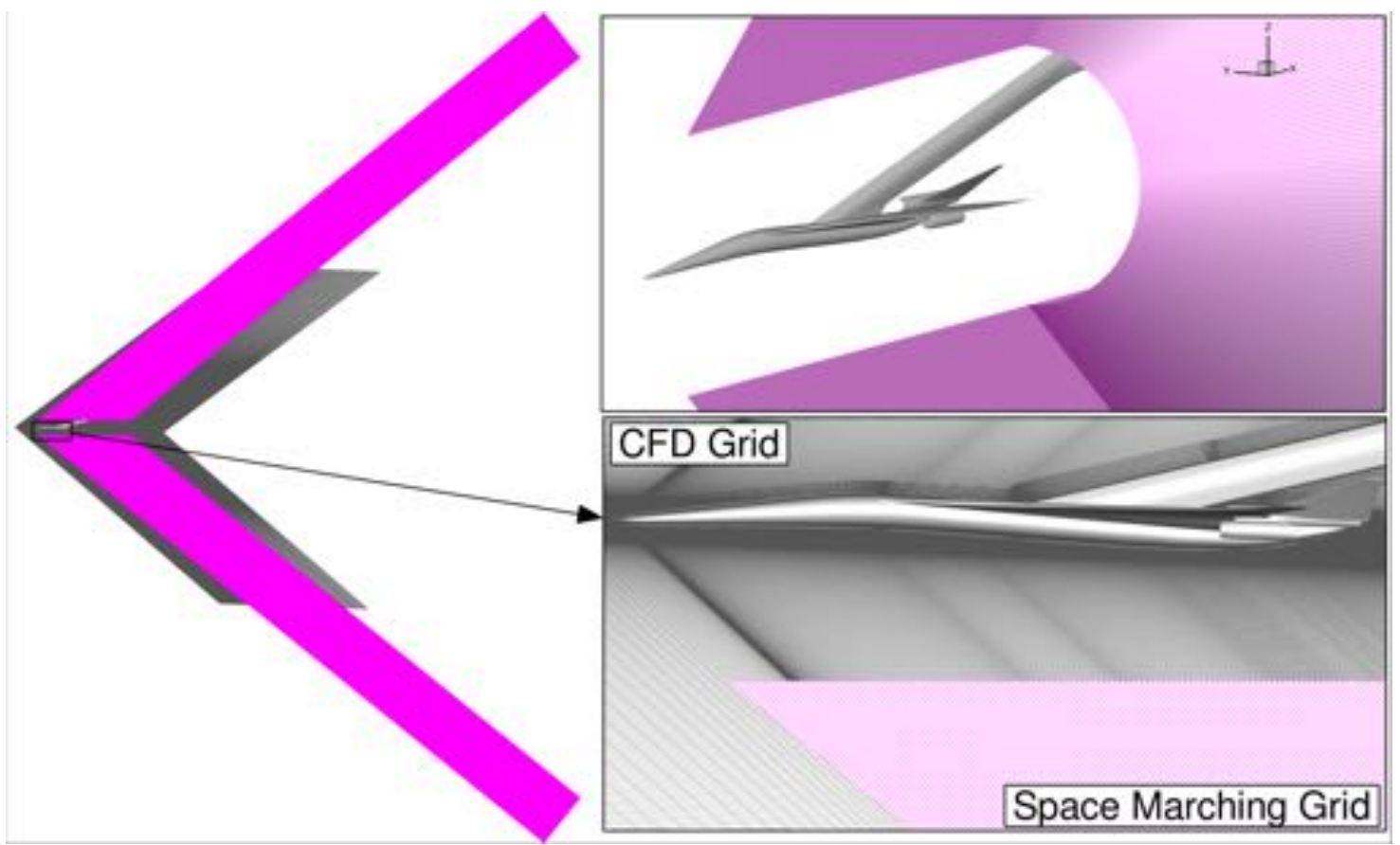

Figure 29. Plot of the automatically generated space marching grid (magenta) over-laying the CFD grid (grey) on the symmetry plane. Inset Top: Isometric view of the space marching grid with the LM-1021 wind tunnel test article. Inset Bottom: Close-up view of the CFD grid and the space marching grid.

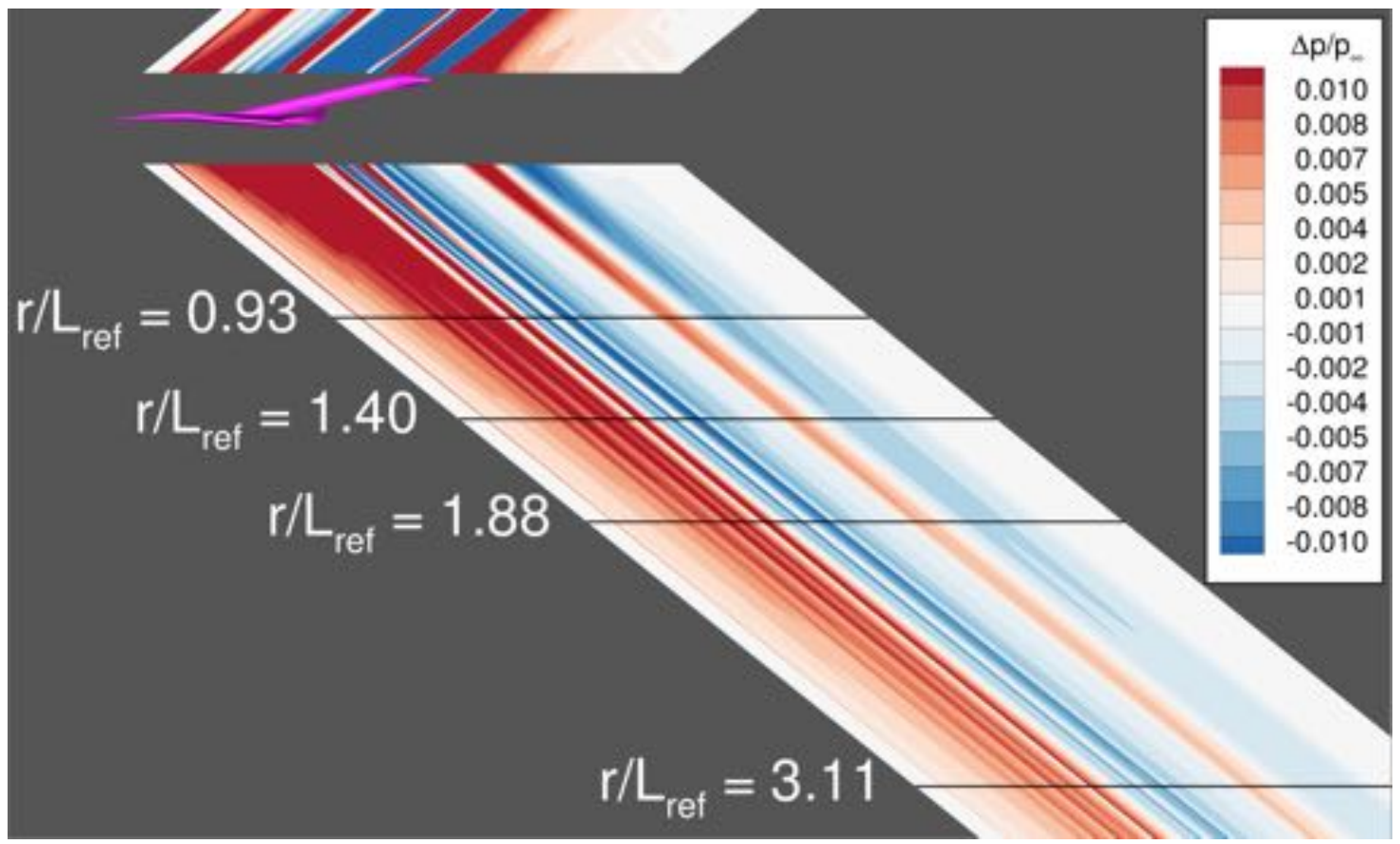

Figure 30. Diagram of the radial extraction locations below the LM-1021 wind tunnel test article including $\mathrm{r} / \mathrm{L}_{\text {ref }}=0.93,1.4,1.88,3.11$ along with pressure contours on the symmetry plane. 


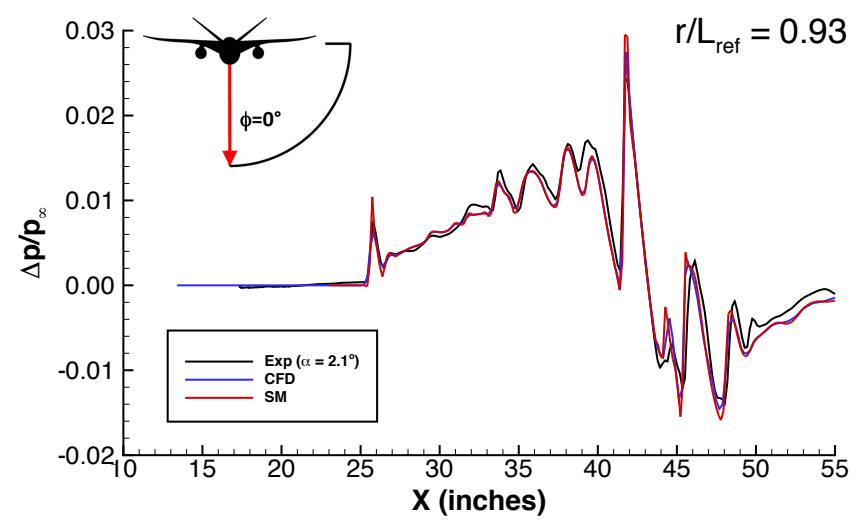

(a)

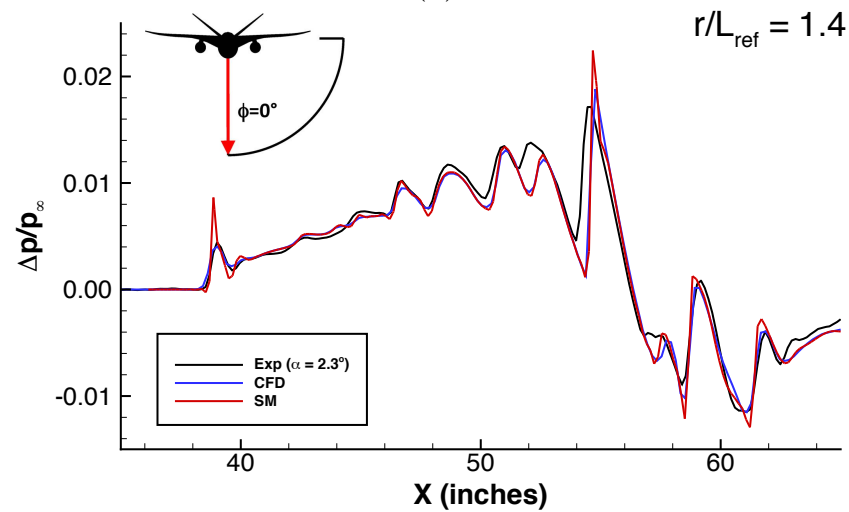

(b)

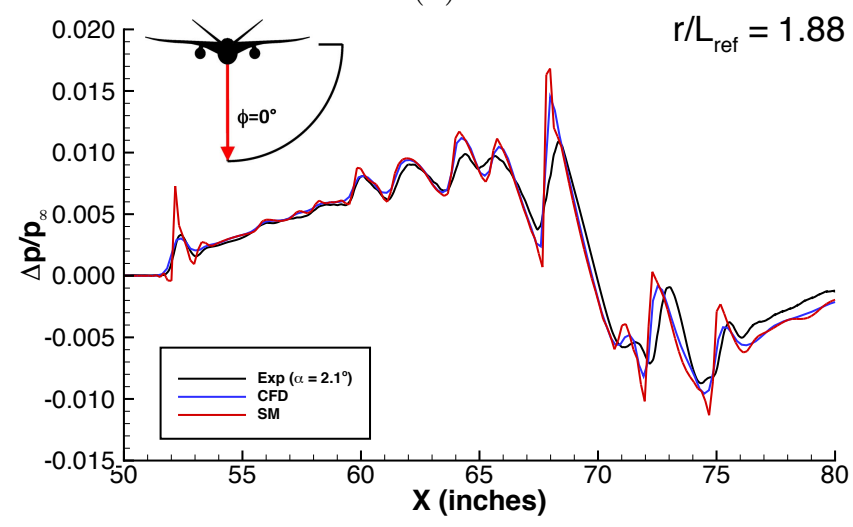

(c)

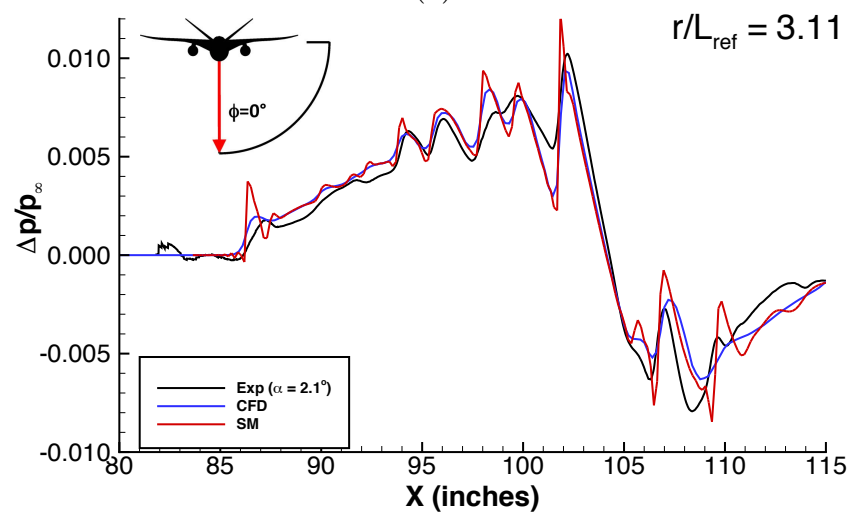

(d)

Figure 31. Comparison of pressure signatures between the space marching solution, the CFD solution, and the wind tunnel measurements at (a) $r / L_{\text {ref }}=0.93$, (b) $r / L_{\text {ref }}=1.4$, (c) $r / L_{\text {ref }}=1.88$, (d) $r / L_{\text {ref }}=3.11$

\section{4 of 34}

